\title{
A mesh-free method with arbitrary- order accuracy for acoustic wave propagation
}

\section{$\operatorname{AUTHOR}(\mathrm{S})$ :}

Takekawa, Junichi; Mikada, Hitoshi; Imamura, Naoto

\section{CITATION:}

Takekawa, Junichi ...[et al]. A mesh-free method with arbitrary-order accuracy for acoustic wave propagation. Computers \& Geosciences 2015, 78: 15-25

\section{ISSUE DATE:}

2015-05

URL:

http://hdl.handle.net/2433/196039

\section{RIGHT:}

(C) 2015 Elsevier Ltd. NOTICE: this is the author's version of a work that was accepted for publication in Computers \& Geosciences. Changes resulting from the publishing process, such as peer review, editing, corrections, structural formatting, and other quality control mechanisms may not be reflected in this document. Changes may have been made to this work since it was submitted for publication. A definitive version was subsequently published in Computers \& Geosciences, 78, 2015, doi:10.1016/j.cageo.2015.02.006; この論文は出版社版でありません。引用の際には出版社版を ご確認ご利用ください。; This is not the published version. Please cite only the published version. 


\section{Title:}

2 A mesh-free method with arbitrary-order accuracy for

3

4

5

6

7

\section{Authors:}

Junichi TAKEKAWA ${ }^{1}$

${ }^{1}$ Department of Civil and Earth Resources Engineering, Kyoto University

e-mail: takekawa@tansa.kumst.kyoto-u.ac.jp

\section{Hitoshi MIKADA ${ }^{1}$}

${ }^{1}$ Department of Civil and Earth Resources Engineering, Kyoto University Naoto IMAMURA ${ }^{1}$

${ }^{1}$ Department of Civil and Earth Resources Engineering, Kyoto University

\section{Corresponding author:}

Junichi TAKEKAWA

Department of Civil and Earth Resources Engineering, Kyoto University

e-mail: takekawa@tansa.kumst.kyoto-u.ac.jp

Phone: +81-75-383-3197

Postal address: C1-1-111, Kyotodaigaku-Katsura, Nishikyo-ku, Kyoto, Japan 
1

2

\section{Abstract:}

In the present study, we applied a novel mesh-free method to solve acoustic wave equation.

Although the conventional finite difference methods determine the coefficients of its operator based on the regular grid alignment, the mesh-free method is not restricted to regular arrangements of calculation points. We derive the mesh-free approach using the multivariable Taylor expansion. The methodology can use arbitrary-order accuracy scheme in space by expanding the influence domain which controls the number of neighboring calculation points. The unique point of the method is that the approach calculates the approximation of derivatives using the differences of spatial variables without parameters as e.g. the weighting functions, basis functions. Dispersion analysis using a plane wave reveals that the choice of the higher-order scheme improves the dispersion property of the method although the scheme for the irregular distribution of the calculation points is more dispersive than that of the regular alignment. In numerical experiments, a model of irregular distribution of the calculation points reproduces acoustic wave propagation in a homogeneous medium same as that of a regular lattice. In an inhomogeneous model which includes low velocity anomalies, partially fine arrangement improves the effectiveness of computational cost without suffering from accuracy reduction. Our result indicates that the method would provide accurate and efficient solutions for acoustic wave propagation using adaptive 
1 distribution of the calculation points.

2

3 Keywords:

4 Mesh-free method; Acoustic wave propagation; Taylor expansion; Numerical solutions.

5

6 
1

2

\section{INTRODUCTION}

Forward modeling techniques of wave propagation are indispensable tools for the implementation of reverse time migration (RTM) and full waveform inversion (FWI)

(Tarantola, 1984; Virieux et al., 2011). Recently, more complex models like salt dome model (e.g. BP model), which include large velocity contrasts, become a target of FWI (Cha and Shin, 2010). Since the most computationally expensive part of these numerical schemes

is the forward modeling, the computational efficiency is recognized as one of the keys to improve the effectiveness of the schemes. For the calculation of full-waveform synthetic seismic traces in inhomogeneous models, numerical simulation methods such as finite difference (FD) and finite element (FE) have often been used. FD method has been widely used for many years as a simulator of acoustic wave propagation, and highly accurate and efficient FD operators developed by many researchers (e.g. Virieux, 1986; Chu and Stoffa, 2012; Liu et al., 2014a; Liu et al., 2014b; Tan and Huang, 2014) are available. These schemes are compared to each other in terms of the numerical accuracy and computational efficiency (e.g. Liang et al., 2014). In many cases, the coefficients of FD operators are derived based on the regular lattice grids. To overcome problems that may arise to handle arbitrary shaped anomalies or topographies using the regular lattice grids, curvilinear schemes for modeling wave propagation have been developed (e.g. Tarrass et al., 2011). Although these schemes 
1 can handle arbitrary shaped topography, arrangement of optimal grid for complex velocity

2 models is not straightforward. On the other hand, FE method uses numerical meshes to

3 build arbitrary shaped models. The method provides the flexibility and the accuracy in the

4 calculation through the mesh generation process, which is computationally costly. It is

5 meaningful to have other methods that could deal with non-flat surface or interfaces with less

6 computational load than FE method.

$7 \quad$ Some novel approaches based on a mesh-free concept have also been developed. This

8 class of numerical methods can discretize models of analysis, which include complex

9 topography and/or complex velocity structure, without any mesh structure or regular lattice

10 grids, and use a set of calculation points surrounding each target point for the discretization

11 (e.g. Lee et al., 2003). Wittke and Tezkan (2014) presented a new approach for

12 magnetotelluric modeling using the Meshless Local Petrov-Galerkin method. Wenterodt

13 and Estorff (2009) investigated the dispersion property of the meshfree radial point

14 interpolation method (RPIM) for the Helmholtz equation, and showed a significant reduction

15 of the dispersion error compared with the FE method. The method, however, requires

16 background meshes to conduct the numerical integration. Furthermore, we need to define

17 not only the radius of the influence domain but also the weighting and basis functions.

18 These miscellaneous parameters lead to the complexity in the choice of optimal combination 
1 for minimizing the dispersion error (Wenterodt and Estorff, 2011).

2 O’Brien and Bean (2011) developed an irregular lattice method for elastic wave

3 propagation based on an elastic lattice method (Monette and Anderson, 1994; Toomey and

4 Bean, 2000; O’Brien and Bean, 2004). They overcame the restrictions on the regular lattice

5 through the augmentation in the number of the nearest neighbor points. Takekawa et al.

6 (2012) proposed a particle method to simulate seismic wave propagation induced by

7 earthquakes. The method can introduce free-surface condition just by removing or ignoring

8 any particles above the surfaces, and could be applied to computational rock physics problems

9 (Takekawa et al., 2014a). These methods do not require the background meshes for the

10 numerical integration, and could be classified as true mesh-free methods. However, the

11 methods do not improve the order of the accuracy in space even if the number of neighbors is

12 increased (Takekawa et al., 2014b; Takekawa et al., 2014c). For the utilization of mesh-free

13 models in the forward simulation, the accuracy of methods to apply to the models needs to be

14 revisited.

15 In this study, we present a mesh-free method for solving acoustic wave propagation that could provide the accuracy of arbitrary order based on the multivariable Taylor expansion

17 (Tamai et al., 2013). The method was originally developed for solving incompressible fluid 
1 The high-order scheme could be applied to irregular distributions of particles successfully

2 without any background meshes, i.e. it is also a true mesh-free method. Since the method

3 was originally designed as a general method for solving partial differential equations, we are

4 able to extend the method to solve the acoustic wave equation. The feature of the method is

5 that the approximation of derivatives is calculated by using the differences of spatial variables

6 without parameters as e.g. the weighting functions, basis functions. In other words, the

$7 \quad$ method is a mesh-free FD method. This feature of the method eliminates the complicated

8 process of parameter optimization (Wenterodt and Estorff, 2011).

9 In the present study, we first introduce the basic concept of the method followed by the

10 verification of the dispersion property for both regular and irregular arrangements of

11 calculation points. We then calculate acoustic wave propagation using a homogeneous

12 model with random distribution of calculation points. Finally, we demonstrate the

13 effectiveness of the method using an inhomogeneous model and confirm that our method

14 would be a true mesh-free method where the accuracy can be quantitatively measured.

\section{METHOD}

In this chapter, we explain the basic concept of the mesh-free method based on the 
$1 \quad \underline{f}(\mathbf{r})$ to $M$-th order at position $\mathbf{r}_{\mathrm{i}}$ is expressed as follows;

2

$3 \quad \mathrm{f}\left(\mathbf{r}_{\mathrm{i}}+\Delta \mathbf{r}\right)=\mathrm{f}\left(\mathrm{r}_{\mathrm{i}}\right)+\frac{1}{1 !}\left(\Delta \mathrm{r}_{1} \frac{\partial}{\partial \mathrm{r}_{1}}+\cdots+\Delta \mathrm{r}_{\mathrm{d}} \frac{\partial}{\partial \mathrm{r}_{\mathrm{d}}}\right) \mathrm{f}\left(\mathbf{r}_{\mathrm{i}}\right)+\frac{1}{2 !}\left(\Delta \mathrm{r}_{1} \frac{\partial}{\partial \mathrm{r}_{1}}+\cdots+\Delta \mathrm{r}_{\mathrm{d}} \frac{\partial}{\partial \mathrm{r}_{\mathrm{d}}}\right)^{2} \mathrm{f}\left(\mathbf{r}_{\mathrm{i}}\right)+$

$4 \quad \cdots+\frac{1}{\mathrm{M} !}\left(\Delta \mathrm{r}_{1} \frac{\partial}{\partial \mathrm{r}_{1}}+\cdots+\Delta \mathrm{r}_{\mathrm{d}} \frac{\partial}{\partial \mathrm{r}_{\mathrm{d}}}\right)^{\mathrm{M}} \mathrm{f}\left(\mathbf{r}_{\mathrm{i}}\right)+\mathrm{O}\left(\|\Delta \mathbf{r}\|^{\mathrm{M}+1}\right)$

where $\mathbf{r}_{\mathrm{i}}$ and $\mathbf{r}_{\mathrm{i}}+\Delta \mathbf{r}$ are the position vectors of calculation point $\mathrm{i}$ and its neighboring point $\mathrm{j}$,

$7 \quad \mathrm{~d}$ is the number of spatial dimension. $\quad$ In many cases related to wave propagation, $\mathrm{d}$ may be 2

8 or $3 . \quad \Delta \mathbf{r}$ is the relative position vector between points $\mathrm{i}$ and $\mathrm{j} . \Delta \mathrm{r}_{\mathrm{d}}$ means d-th component

9 of vector $\Delta \mathbf{r}$ (in two-dimensional case, $\left.\Delta \mathbf{r}=\left(\Delta \mathrm{r}_{1}, \Delta \mathrm{r}_{2}\right)\right)$.. We replace $\mathrm{f}\left(\mathbf{r}_{\mathrm{i}}\right)$ and

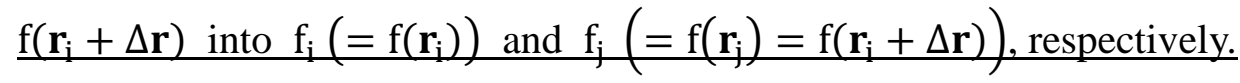

$11 \quad$ Here, we define vectors $\mathbf{P}$ and $\boldsymbol{\delta}$ as follows;

$\mathbf{P}=\left(\Delta \mathrm{r}_{1}, \cdots, \Delta \mathrm{r}_{\mathrm{d}}, \frac{1}{2 !} \Delta \mathrm{r}_{1}^{2}, \Delta \mathrm{r}_{1} \Delta \mathrm{r}_{2}, \cdots, \frac{1}{2 !} \Delta \mathrm{r}_{\mathrm{d}}^{2}, \cdots, \frac{1}{(\mathrm{M}-1) ! 1 !} \Delta \mathrm{r}_{\mathrm{d}-1} \Delta \mathrm{r}_{\mathrm{d}}^{\mathrm{M}-1}, \frac{1}{\mathrm{M} !} \Delta \mathrm{r}_{\mathrm{d}}^{\mathrm{M}}\right)^{\mathrm{T}}$

$$
\boldsymbol{\delta}=\left(\frac{\partial}{\partial \mathrm{r}_{1}}, \cdots, \frac{\partial}{\partial \mathrm{r}_{\mathrm{d}}}, \frac{\partial^{2}}{\partial \mathrm{r}_{1}^{2}}, \frac{\partial^{2}}{\partial \mathrm{r}_{1} \partial \mathrm{r}_{2}}, \cdots, \frac{\partial^{2}}{\partial \mathrm{r}_{\mathrm{d}}^{2}}, \cdots, \frac{\partial^{\mathrm{M}}}{\partial \mathrm{r}_{\mathrm{d}-1} \partial \mathrm{r}_{\mathrm{d}}^{\mathrm{M}-1}}, \frac{\partial^{\mathrm{M}}}{\partial \mathrm{r}_{\mathrm{d}}^{\mathrm{M}}}\right)^{\mathrm{T}}
$$

Vectors $\mathbf{P}$ and $\boldsymbol{\delta}$ include coefficients and derivatives, respectively. Using Eq.(2) and (3), we

transform Eq.(1) as follows; 
$2 \quad\{(\mathbf{P} \cdot \boldsymbol{\delta}) \mathrm{f}\}_{\mathbf{r}=\mathbf{r}_{\mathrm{i}}}=\Delta \mathrm{f}_{\mathrm{ij}}+\mathrm{O}\left(\|\Delta \mathbf{r}\|^{\mathrm{M}+1}\right)$

4 where $\Delta \mathrm{f}_{\mathrm{ij}}=\mathrm{f}_{\mathrm{j}}-\mathrm{f}_{\mathrm{j}}$. Multiplying both sides of Eq.(4) by $\mathbf{P}$, we obtain;

5

$6 \quad\{(\mathbf{P} \cdot \boldsymbol{\delta})(\mathbf{P f})\}_{\mathbf{r}=\mathbf{r}_{\mathrm{i}}}=\mathbf{P} \Delta \mathrm{f}_{\mathrm{ij}}+\mathbf{P} \cdot \mathrm{O}\left(\|\Delta \mathbf{r}\|^{\mathrm{M}+1}\right)$

$7 \Rightarrow(\mathbf{P} \otimes \mathbf{P}) \cdot(\boldsymbol{\delta} \mathbf{f})_{\mathbf{r}=\mathbf{r}_{\mathrm{i}}}=\mathbf{P} \Delta \mathrm{f}_{\mathrm{ij}}+\mathbf{P} \cdot \mathrm{O}\left(\|\Delta \mathbf{r}\|^{\mathrm{M}+1}\right)$

$9 \quad$ Where $\mathbf{a} \otimes \mathbf{b}$ means the tensor product of vectors $\mathbf{a}$ and $\mathbf{b}$.

10 Here, we introduce an influence domain which supports a finite region around $\mathbf{r}=\mathbf{r}_{\mathbf{i}}$.

11 This domain controls the number of neighboring calculation points. Wider range of the

12 influence domain contains larger number of calculation points. The effect of the influence

13 domain on the accuracy and calculation time is investigated in the following section. We

14 calculate the sum of both sides of Eq.(5) inside the influence domain.

$17 \quad(\boldsymbol{\delta} \mathbf{f})_{\mathbf{r}=\mathbf{r}_{\mathrm{i}}} \approx\left\{\sum_{\mathrm{j}}^{\mathrm{n}}(\mathbf{P} \otimes \mathbf{P})\right\}^{-1} \cdot\left\{\sum_{\mathrm{j}}^{\mathrm{n}}\left(\mathbf{P} \Delta \mathrm{f}_{\mathrm{ij}}\right)\right\}$ 
$2 \underline{\mathrm{n} \text { is the number of neighboring calculation points inside the influence domain. Vector }(\delta \mathrm{f}) \text { in }}$

3 Eq.(7) includes derivatives of $f(\mathbf{r})$ at $\mathbf{r}=\mathbf{r}_{\mathrm{i}}$. The size of the matrix $\mathbf{P} \otimes \mathbf{P}$ depends only

4 on the order of accuracy M. For example, in two-dimensional case, the size are $5 \times 5$ and

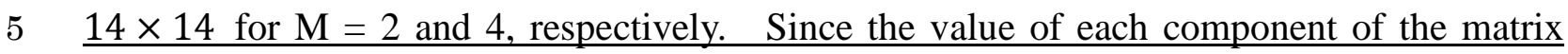

6 depends only on the relative positions between point $\mathrm{i}$ and neighboring points $\mathrm{j}$, the inverse of

$7 \quad$ the matrix can be calculated before starting time steps. Once the inverse at each calculation

8 point is fixed, we continue to use it during the calculation. This means that solving inverse

9 matrices, which is a challenging procedure, can be excluded from the time loop.

Since Eq.(7) is derived under assumption of arbitrary arrangement of the position of $j$, we

can estimate the derivatives of $\mathrm{f}(\mathbf{r})$ at $\mathbf{r}=\mathbf{r}_{\mathrm{i}}$ in a mesh-free framework. The calculation

only requires the positions of calculation points and the differences between $f_{i}$ and $f_{j}$, i.e. it is a

14 the inverse matrix in Eq.(7) can be solved.

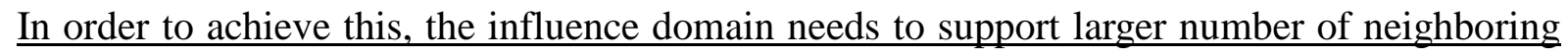


1 calculation points than the number of terms in Eq.(2) and (3). The minimum radii of the

2 influence domain for the regular lattice alignment with different $\mathrm{M}$ are shown in Fig.1. It is

3 also noted that particular distributions of neighboring points (e.g. in two-dimensional case,

4 neighbors line up in a linear arrangement) do not satisfy Eq.(8) even if the number of

$5 \quad$ neighboring points is sufficient.

6 The solution vector $\boldsymbol{\delta}$ f includes from first order to M-th order derivatives of $\mathrm{f}(\mathbf{r})$ at $\mathbf{r}=$

$7 \quad \mathbf{r}_{\mathrm{i}}$. These components can be obtained by solving Eq.(7). However, it is needed to

8 calculate only second derivatives of $\mathrm{f}(\mathbf{r})$ for solving the acoustic wave equation (in the

9 two-dimensional case, $\partial^{2} \mathrm{f}(\mathbf{r}) / \partial \mathrm{r}_{1}^{2}$ and $\left.\partial^{2} \mathrm{f}(\mathbf{r}) / \partial \mathrm{r}_{2}^{2}\right)$. We only have to calculate the

10 required components for solving acoustic wave equation in Eq.(7).

11 Here we refer to the boundary conditions (Dirichlet and Neumann). Dirichlet condition,

12 for example a free boundary condition in acoustics, is implemented at the free surface (e.g.

13 sea surface) directly. Thus, the pressure of calculation points at the free surface is set to zero.

14 This type of boundary condition is simply applied to arbitrary shaped surface. The

15 implementation of Neumann condition, however, is not straightforward because determination

16 of a vector normal to boundaries for arbitrary shaped surface takes a little ingenuity (e.g.

17 Nomura et al., 2001). Although it is simple to implement Neumann condition using known

18 normal vectors because the first derivation is also calculated by Eq.(7), tackling this problem 
1 is beyond to our purpose of this study.

2 FD schemes with high-order in time have also been proposed (e.g. Chen, 2011). However,

3 we use only second-order in time because the purpose of this study focus on the development

4 of the mesh-free approach in the space. Applying the second-order scheme in time to the

5 acoustic wave equation and considering two-dimensional in space, we can obtain the

6 following equation;

7

$8 \quad \frac{p_{i}^{t+1}-2 p_{i}^{t}+p_{i}^{t-1}}{\Delta t^{2}}=v\left(r_{i}\right)^{2}\left\{\frac{\partial^{2}}{\partial r_{1}^{2}} p^{t}+\frac{\partial^{2}}{\partial r_{2}^{2}} p^{t}\right\}_{r=r_{i}}$

10 where $\mathrm{p}$ is the pressure, subscript $\mathrm{i}$ and superscript $\mathrm{t}$ mean the indices of calculation point and

11 the time step, respectively, $\mathrm{v}$ is the velocity, $\Delta \mathrm{t}$ is the time spacing. The spatial derivatives

12 in the right-hand side of Eq.(9) can be calculated by using Eq.(7). The pressure can be

13 updated as follows;

$15 \quad \mathrm{p}_{\mathrm{i}}^{\mathrm{t}+1}=2 \mathrm{p}_{\mathrm{i}}^{\mathrm{t}}-\mathrm{p}_{\mathrm{i}}^{\mathrm{t}-1}+\mathrm{v}\left(\mathbf{r}_{\mathrm{i}}\right)^{2} \Delta \mathrm{t}^{2}\left\{\frac{\partial^{2}}{\partial \mathrm{r}_{1}^{2}} \mathrm{p}^{\mathrm{t}}+\frac{\partial^{2}}{\partial \mathrm{r}_{2}^{2}} \mathrm{p}^{\mathrm{t}}\right\}_{\mathbf{r}=\mathbf{r}_{\mathrm{i}}}$

17 Here, we refer to the spatial order of accuracy of the mesh-free method. In Eq.(6), the 
1 order of the truncation error is $\mathrm{O}\left(\|\Delta \mathbf{r}\|^{\mathrm{M}-\mathrm{k}+1}\right)$, where $\mathrm{M}$ and $\mathrm{k}$ mean the order of accuracy

2 and order of spatial derivatives, respectively. So, the truncation error of the final equation

3 Eq.(10) is also $\mathrm{O}\left(\|\Delta \mathbf{r}\|^{\mathrm{M}-\mathrm{k}+1}\right)$ for arbitrary distributions of calculation points.

\section{DISPERSION ANALYSIS}

We conduct a dispersion analysis to investigate the dispersion property of the method. In this section, we assume regular and irregular lattice arrangement of the calculation points. To make an irregular arrangement, calculation points are migrated using a uniform pseudorandom number from the regular lattice as shown in Fig.2. The spacing of the regular lattice is $\mathrm{l}_{0}$. The migration distance $\Delta \mathrm{l}_{0}$ and angle $\theta$ are decided in a random manner while the source and receiver positions are fixed. The maximum migration distance is $l_{0} / 4$. This means that the minimum and maximum spacing of calculation points are $0.5 \times \mathrm{l}_{0}$ and

$131.5 \times l_{0}$, respectively. We consider a plane wave of the form $p=p_{0} \exp (-i \omega t+i k x)$

14 propagating along the $\mathrm{x}$-axis with wavenumber $\mathrm{k}$ and frequency $\omega$. The model is assigned a P-wave velocity of $3500 \mathrm{~m} / \mathrm{s}$ with the regular spacing set to $10 \mathrm{~m}$. For the second-order

17 follows; 
$1 \quad \frac{\partial^{2} \mathrm{p}}{\partial \mathrm{t}^{2}}=-\omega^{2} \mathrm{p}_{0} \exp (-\mathrm{i} \omega \mathrm{t}+\mathrm{ikx})$

2

3 The effect of approximation of time derivative is investigated in the following. Substituting

4 the plane wave equation into Eq.(10) derives the dispersion property of the method (details

5 are shown in Appendix A).

6 Fig.3. shows the relationships between $\mathrm{k}$ and $\omega$ for the case of $M=2,4,6$ and 8 using the

7 regular lattice. The number of calculation points in the influence domain for each case is

8 same as shown in Fig.1. The dispersion curves are in absolute agreement with those from

9 FD operators derived from the Taylor expansion (e.g. Chu and Stoffa, 2012) if we use the regular arrangement.

Fig.4 shows the dispersion curves for the irregular arrangement in case of $\mathrm{M}=2$ and 4 .

We show some curves for different sizes of the influence domain. In other words, we use

different number $\mathrm{n}$ in Eq.(7) for calculating the spatial derivatives without changing the order

14 of accuracy M. For the irregular arrangement, the case for smaller number of calculation

15 points induces large misfit ( $n=8$ in Fig.4 (a) and $n=20$ in Fig.4 (b)). The misfit decreases

16 with increasing the number of calculation points ( $n=12$ and 20 in Fig.4 (a) and $n=28$ and 36

17 in Fig.4 (b)). We can see little change in the dispersion curves if the radius of the influence domain reaches a certain size. The above results suggest that sufficient large size of the 
1 influence domain is recommended to avoid numerical dispersion whereas oversized domain

2 should be avoided because the large number of calculation points increases the computational

3 cost. Calculation time is strongly dependent on the number of neighboring points. For

4 effective calculations, adequate number of neighboring points is needed especially in

5 three-dimensional cases. We will investigate the calculation time in terms of the number of

6 neighboring points in the following section. Comparing the dispersion curve for the regular

7 arrangement with the irregular arrangement, it is more dispersive for the same number of

8 calculation points per wavelength. If we use higher order scheme (i.e. larger number of $\mathrm{M}$ in

9 Eq.(2) and (3)), the dispersion property can be improved for both regular and irregular

10 arrangements.

11 We next investigate the effect of the time step on the dispersion property. The second

12 order finite difference approximation is used for time derivative instead of Eq.(11).

$14 \quad \frac{\partial^{2} \mathrm{p}}{\partial \mathrm{t}^{2}} \approx \frac{\mathrm{p}(\mathrm{t}+\Delta \mathrm{t})-2 \mathrm{p}(\mathrm{t})+\mathrm{p}(\mathrm{t}-\Delta \mathrm{t})}{\Delta \mathrm{t}^{2}}$.

17 step is normalized by a maximum CFL parameter $\alpha_{\max }\left(=\mathrm{v} \cdot \Delta \mathrm{t}_{\max } / \Delta \mathrm{x}\right) . \Delta \mathrm{t}_{\max }$ means

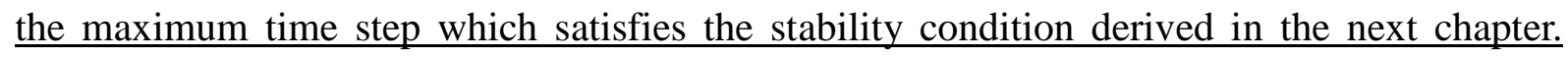


1 The result shows that the time step has the influence on the dispersion property same as other

2 numerical simulators. Although the errors of each curve differ from each other especially in

3 small number of calculation points per a wave length, there is little difference in small wave

4 number region.

\section{STABILITY CONDITION}

In the acoustic modeling, fine spatial grid or coarse time step may trigger exponential increment of the amplitude. Grid spacing and time step should be determined so that schemes can avoid the unstable condition. In this chapter, we investigate the stability condition of the mesh-free method. Following the previous study about stability discussions

11 for the scalar wave equation (e.g. Wu et al., 1996; Lines et al., 1999), we derive the maximum

12 time step for the stable calculation using the CFL parameter $\alpha(=\mathrm{v} \cdot \Delta \mathrm{t} / \Delta \mathrm{x})$. $\quad \mathrm{v}$ is the maximum velocity in the model. $\Delta \mathrm{x}$ is the minimum spacing between the calculation

14 points.

15 Fig.6 (a) shows the maximum allowed number $\alpha$ for the mesh-free method with different

16 order M. The number of neighboring calculation points is the same as Fig.1. On the other

17 hand, Fig.6 (b) shows the result with the different number of calculation points $\mathrm{n}$ in case of $\mathrm{M}$

$18 \equiv 2$. Vertical axis shows the maximum CFL parameter $\alpha_{\max }$ for the stable calculation. 
1 The stability condition becomes less stringent with increasing the order of accuracy, whereas

2 larger number of calculation points can relax the stability condition.

3 To confirm this result, we conduct two numerical experiments with different time steps, i.e.

4 a) $\Delta t=\Delta t_{\max }$, b) $\Delta t=1.01 \times \Delta t_{\max }$. We set to $v=2000 \mathrm{~m} / \mathrm{s}, \Delta \mathrm{x}=10 \mathrm{~m}, \mathrm{M}=2$ and $\mathrm{n}$

$5=8$. In this condition, $\Delta \mathrm{t}_{\max }$ equals to 0.005 . Fig.7 shows snapshots with different $\Delta \mathrm{t}$

6 after 200 time steps. An artificial wave field as sort of checkerboard pattern can be observed

$7 \quad$ only in Fig.7 (b). It should be noted that the scale of contour are quite different from each

8 other. Before reaching three-hundredth time step, the calculation was terminated in case b).

9 This result indicates that the numerical condition changed from stability to instability in $\Delta \mathrm{t}=$

$\underline{1.01 \times \Delta \mathrm{t}_{\max }}$.

\section{NUMERICAL EXAMPLES}

\subsection{Effect of spatial order}

We investigate the accuracy of our method comparing it with an analytical solution using a

15 two-dimensional homogeneous medium which has a P-wave velocity of $2000 \mathrm{~m} / \mathrm{s}$. The

16 model consists of a regular lattice alignment of calculation points with a constant spacing of

$1710 \mathrm{~m}$. The source is set at the center of the model. The waveform of the source is the

18 Ricker wavelet with a central frequency of $18 \mathrm{~Hz}$. In this condition, the number of 
1 calculation points in a minimum wavelength is about 4.04. A receiver with the offset

2 distance of about $2263 \mathrm{~m}$ is selected. This means that the receiver is located at

3 approximately 56 times the minimum wavelength. We calculate acoustic wave propagation

4 using our method with different-order schemes.

$5 \quad$ Fig.8 shows the snapshots calculated by the schemes of $M=2,4,6$ and 8 after 1.75 s. A

6 circle and a triangle represent the source and receiver positions. In the case of $M=2$, severe

7 numerical dispersion can be observed. The scheme of $\mathrm{M}=4$ represents a great improvement

8 compared with $\mathrm{M}=2$ while a minor degree of dispersion still remains. For schemes of $\mathrm{M}=$

96 and 8, the dispersion is suppressed almost completely. Since the number of calculation

10 points per minimum wavelength is about 4.04 (i.e. $\Delta x / \lambda \approx 0.25$ ), the result has good

11 agreement with the result from the dispersion analysis in the previous. Fig.9 shows the

15

$\mathrm{p}(\mathrm{x}, \mathrm{z}, \mathrm{t})=\mathrm{i} \pi \mathrm{F}^{-1}\left(\mathrm{H}_{0}^{(2)}(\mathrm{kR}) \mathrm{F}(\mathrm{f}(\mathrm{t}))\right)$

17 where $F(\cdot)$ and $F^{-1}(\cdot)$ represent forward and inverse Fourier transformations, respectively,

$18 \mathrm{f}(\mathrm{t})$ is time series of the source, $\mathrm{H}_{0}^{(2)}(\cdot)$ is the second Hankel function of order zero, $\mathrm{k}$ is the 
1 wavenumber $(=\omega / v)$, and $R$ is the distance between the source and the receiver. It is

2 observed that the high-order scheme can suppress the spurious oscillation and has a good

3 agreement with the analytical solution. In this way, our scheme can select arbitrary-order

4 accuracy depending on the numerical conditions (e.g. source frequency, spatial resolution,

5 etc.).

6

$7 \quad 5.2$ Effect of irregular arrangement

8

9 the source is a Ricker wavelet with a central frequency of $5 \mathrm{~Hz}$. In this case, the number of calculation points per minimum wavelength is about 14.4. Four receivers are set with an offset distance of about $566 \mathrm{~m}$. In this section, we use the scheme of $\mathrm{M}=4$ in space. The radius of the influence domain is set to $3.6 \times \mathrm{l}_{0}$ to support sufficient number of calculation points. The average number of calculation points in the influence domain is about 36 . We also simulate acoustic wave propagation using the regular lattice alignment for comparison. 
1 arrangement. A circle and triangles represent the source and receivers, respectively. Fig.10

2 (b) shows the close-up figure around the acoustic wave front marked with a dotted square in

Fig.10 (a). It can be observed that the pressure field is reproduced smoothly by the irregular arrangement. Fig.11 shows the waveforms recorded at the receiver positions with different offsets. Solid gray and dotted black lines represent waveforms calculated by the regular and irregular arrangements, respectively. Since both waveforms have good agreement with each other, the validity of our mesh-free method for irregular arrangement is ensured.

\subsection{Wave propagation in an inhomogeneous medium}

Finally, we demonstrate the effectiveness of the method using a simple inhomogeneous model as shown in Fig.12. A low velocity anomaly exists at the center of the model. The velocity of the anomaly is half of the surrounding area. The source is located at the left side of the anomaly. The four receivers are set with $400 \mathrm{~m}$ intervals. The second and third receivers are located inside the anomaly. We calculate wave propagation using three different arrangements, a) fine arrangement, b) coarse arrangement, and c) partially fine arrangement (Fig.13). In the partially fine arrangement case, the finer calculation points are used only around the low velocity anomaly.

$\underline{\text { In FD method, we can introduce partially fine resolution using the discontinuous grids on }}$ 
1 the boundary of the spatial resolution (e.g. Aoi and Fujiwara, 1999). However, this requires

2 some special treatment when waves propagate through small and large grids. This

3 complexifies the application of it on arbitrary velocity structures. In FE methods, we need a

4 re-meshing which includes a time consuming process especially for complex velocity models.

5 On the other hand, in the mesh-free method, we do not require the implementation of the

6 discontinuous grids or a re-meshing process.

7 The waveform of the source is the Ricker wavelet with a central frequency of $9 \mathrm{~Hz}$. In

8 this case, the number of calculation points in a minimum wavelength is about 8.08 or 4.04 for

9 the fine and course arrangements, respectively.

If we use the same radius of the influence domain for fine and coarse regions, the number

11 of calculation points increases uneconomically in the fine region, i.e. increasing

12 computational burden. Therefore, the radius is determined by the grid spacing of each

13 region so that each domain supports minimal number of neighbors as shown in Fig.1. This

14 means that the radius in the fine region is half of that in the coarse region. At the interface

15 of the different spatial resolution, the radius of the influence domain is determined by the

16 averaged spacing (Eq. (2) and (3) in Takekawa et al., 2012).

17 Fig.14 shows the snapshots of the pressure field after 1 and $1.25 \mathrm{~s}$ using different

18 arrangements. If we use the coarse arrangement (Fig.14 (b) and (e)), the numerical 
1 dispersion is occurred as indicated by arrows. On the other hand, for the fine and partially

2 fine arrangements, the dispersion cannot be observed. Fig.15 shows the waveforms

3 observed at the receivers. The results are compared with that from FEM which can

4 reproduce curved surfaces accurately. The waveforms calculated by the fine and partially

5 fine arrangements have good agreement with that from FEM whereas the waveform

6 calculated by the coarse ones are quite different from the reference waveforms due to

7 numerical dispersion. If we use the coarse arrangement, the number of calculation points per

8 minimum wavelength inside the anomaly is same as that in section 4.1. In Fig.8 and 9, we

9 can observe a slight dispersion for the scheme of $M=4$. This means that the number of

10 calculation points of the coarse arrangement was not sufficient to resolve the low velocity

11 anomaly.

The above results indicate that the partially fine arrangement can provide accurate results

13 in an efficient manner by introducing appropriate spacing of the calculation points which is

14 suitable for arbitrary velocity structures. Since the method is based on the mesh-free concept, the procedure for the implementation of discontinuous grids or re-meshing is not required. This advantage would improve the numerical accuracy and efficiency in a simple manner, and reduce time for pre-processing. 


\section{CALCULATION TIME}

We compare the calculation time of the mesh-free method with that of the conventional

FDM (e.g. Chu and Stoffa, 2012). The numerical model, which consists of $400 \times 400$

grids or calculation points, is homogeneous with a P-wave velocity of $2000 \mathrm{~m} / \mathrm{s}$. In the

mesh-free method, a regular lattice alignment is used. $2^{\text {nd }}, 4^{\text {th }}, 6^{\text {th }}$ and $8^{\text {th }}$ order schemes for

$6 \quad$ FDM and $M=2,4,6$ and 8 for the mesh-free method are tested. Fig.16 (a) shows the

7 calculation time normalized by that from the $2^{\text {nd }}$ order FDM. In each order of accuracy, the

8 present method costs two to three times more than FDM as is the case in other mesh-free

9 methods. This indicates that FDM has an advantage in the calculation time over the

10 mesh-free method if the numerical model is homogeneous or smooth. On the other hand, the

11 mesh-free method would gain an edge on structured grids if models include large velocity

12 contrast like shown in Fig.12.

Fig.16 (b) shows the effect of the number of calculation points on the calculation time.

14 The order of accuracy $\mathrm{M}$ is fixed to 4. This result indicates that the calculation time strongly

15 depends on the number of calculation points in the influence domain. It is suggested that the

16 oversized domain should be avoided for efficient implementation.

17 The superiority of the mesh-free method depends on the model complexity. Therefore,

18 the method used should be selected for individual cases. The mesh-free method would work 
1 well for models with large velocity contrast, especially in three-dimensional cases. The

2 comparison of the calculation time in Fig.16 provides an indication of the selection of the

3 method.

\section{CONCLUSIONS AND PERSPECTIVES}

6

7 homogeneous model, we used regularly and irregularly distributed calculation points. The numerical results calculated from both the distributions showed good agreement with each other. In the inhomogeneous case, we demonstrated the effectiveness of the method using different resolutions i) fine resolution, ii) coarse resolution, and iii) partially fine resolution. The coarse resolution model showed numerical dispersion in a low velocity anomaly zone. 
1 On the other hand, the partially fine resolution model could suppress the dispersion. The

2 computational time of the method is two or three times expensive than FDM. However, we

3 believe that the method can be an alternative of the conventional method if the model includes

$4 \quad$ large velocity contrasts.

$5 \quad$ Since the present method is a general method for solving partial differential equations, not

6 only acoustic wave propagation but also elastic wave propagation could be solved. In the

7 elastic case, variables (e.g. velocity and stress) are often placed alternately. In our method,

8 the calculation of derivatives needs to be given at the position of the variables for which we

9 evaluate the derivatives. Therefore, some sort of invention is required to implement our

10 method to the staggered arrangement of variables.

11 The advantage of the method is that there is no restriction on the arrangement of the calculation points for achieving arbitrary-order accuracy by simply expanding the influence domain (i.e. increasing the number of neighbors). This would enable us to adopt appropriate spatial resolution corresponding to complex velocity structures in arbitrary and simple manners. This advantage could be exploited in not only FWI for complex velocity models (e.g. salt dome models) but also rock physics problems which include complex microstructures and high contrast in the physical properties. 


\section{APPENDIX A}

2 We derive the dispersion relation of the mesh-free method. For simplicity, the case of M

$3=2$ and $\mathrm{n}=8$ is considered. We consider a plane wave of the form $\mathrm{p}=\mathrm{p}_{0} \exp (-\mathrm{i} \omega \mathrm{t}+\mathrm{ikx})$

4 propagating along the $\mathrm{x}$-direction with wavenumber $\mathrm{k}$ and frequency $\omega$. $\mathrm{p}_{0}$ is the amplitude

5 of the plane wave. The target point $\mathrm{i}$ and neighboring points $\mathrm{j}(\mathrm{j}=1 \sim 8)$ are difined as

6 shown in Fig.A.1. In this case, vectors $\mathbf{P}$ in Eq.(2) is shown as follows;

7

$8 \quad \mathbf{P}_{1}=\left\{-\mathrm{l},-\mathrm{l}, \frac{1}{2} \mathrm{l}^{2}, \mathrm{l}^{2}, \frac{1}{2} \mathrm{l}^{2}\right\}^{\mathrm{T}}$

$9 \quad \mathbf{P}_{2}=\left\{-1,0, \frac{1}{2} 1^{2}, 0,0\right\}^{\mathrm{T}}$

$10 \quad \mathbf{P}_{3}=\left\{-\mathrm{l}, \mathrm{l}, \frac{1}{2} \mathrm{l}^{2},-\mathrm{l}^{2}, \frac{1}{2} \mathrm{l}^{2}\right\}^{\mathrm{T}}$

$11 \mathbf{P}_{4}=\left\{0,-1,0,0,0, \frac{1}{2} l^{2}\right\}^{\mathrm{T}}$

$12 \quad \mathbf{P}_{5}=\left\{0,1,0,0,0, \frac{1}{2} \mathrm{l}^{2}\right\}^{\mathrm{T}}$

$13 \quad \mathbf{P}_{6}=\left\{\mathrm{l},-\mathrm{l}, \frac{1}{2} \mathrm{l}^{2},-\mathrm{l}^{2}, \frac{1}{2} \mathrm{l}^{2}\right\}^{\mathrm{T}}$

$14 \quad \mathbf{P}_{7}=\left\{\mathrm{l}, 0,0, \frac{1}{2} \mathrm{l}^{2}, 0,0\right\}^{\mathrm{T}}$

$15 \quad \mathbf{P}_{8}=\left\{\mathrm{l}, \mathrm{l}, \frac{1}{2} \mathrm{l}^{2}, \mathrm{l}^{2}, \frac{1}{2} \mathrm{l}^{2}\right\}^{\mathrm{T}}$

17 where $\mathrm{l}$ is the spacing between calculation points as shown in Fig.A.1. Subscript is index for 
1 neighboring calculation points. Using Eqs.(A-1), we can calculate $\mathbf{P}_{\mathbf{j}} \otimes \mathbf{P}_{\mathbf{j}}$ and the sum of

2 them.

3

$4 \quad \sum_{j=1}^{8}\left(\mathbf{P}_{j} \otimes \mathbf{P}_{j}\right)=\left\{\begin{array}{ccccc}61^{2} & 0 & 0 & 0 & 0 \\ 0 & 61^{2} & 0 & 0 & 0 \\ 0 & 0 & \frac{3}{2} 1^{2} & 0 & 1^{4} \\ 0 & 0 & 0 & 41^{4} & 0 \\ 0 & 0 & 1^{4} & 0 & \frac{3}{2} l^{2}\end{array}\right\}$

5

$6 \quad$ We also calculate the inverse of Eq.(A.2) as follows.

7

$8 \quad\left\{\sum_{j=1}^{8}\left(\mathbf{P}_{j} \otimes \mathbf{P}_{j}\right)\right\}^{-1}=\left\{\begin{array}{ccccc}\frac{1}{61^{2}} & 0 & 0 & 0 & 0 \\ 0 & \frac{1}{61^{2}} & 0 & 0 & 0 \\ 0 & 0 & \frac{6}{51^{4}} & 0 & -\frac{4}{51^{4}} \\ 0 & 0 & 0 & \frac{1}{41^{4}} & 0 \\ 0 & 0 & -\frac{4}{51^{4}} & 0 & \frac{6}{51^{4}}\end{array}\right\}$

9

10 Differences of pressure between the calculation point $\mathrm{i}$ and neighboring points $\mathrm{j}$ are

11 calculated using a plane wave equation.

12

13

$$
\mathrm{p}_{1}-\mathrm{p}_{\mathrm{i}}=\{\exp (-\mathrm{ikl})-1\} \mathrm{p}_{0} \exp (-\mathrm{i} \omega \mathrm{t}+\mathrm{ikx})
$$


$1 \quad \mathrm{p}_{2}-\mathrm{p}_{\mathrm{i}}=\{\exp (-\mathrm{ikl})-1\} \mathrm{p}_{0} \exp (-\mathrm{i} \omega \mathrm{t}+\mathrm{ikx})$

$2 \quad \mathrm{p}_{3}-\mathrm{p}_{\mathrm{i}}=\{\exp (-\mathrm{ikl})-1\} \mathrm{p}_{0} \exp (-\mathrm{i} \omega \mathrm{t}+\mathrm{ikx})$

$3 \quad \mathrm{p}_{4}-\mathrm{p}_{\mathrm{i}}=0$

$4 \quad p_{5}-p_{i}=0$

$5 \quad \mathrm{p}_{6}-\mathrm{p}_{\mathrm{i}}=\{\exp (\mathrm{ikl})-1\} \mathrm{p}_{0} \exp (-\mathrm{i} \omega \mathrm{t}+\mathrm{ikx})$

$6 \quad \mathrm{p}_{7}-\mathrm{p}_{\mathrm{i}}=\{\exp (\mathrm{ikl})-1\} \mathrm{p}_{0} \exp (-\mathrm{i} \omega \mathrm{t}+\mathrm{ikx})$

$7 \quad \mathrm{p}_{8}-\mathrm{p}_{\mathrm{i}}=\{\exp (\mathrm{ikl})-1\} \mathrm{p}_{0} \exp (-\mathrm{i} \omega \mathrm{t}+\mathrm{ikx})$

9 Using Eqs. (A.1), (A.3), (A.4) and (7), we can calculate spatial derivatives at the position of

10 target point i.

11

$12 \frac{\partial^{2} \mathrm{p}}{\partial \mathrm{r}_{1}^{2}}=\frac{1}{\mathrm{l}^{2}}(\exp (-\mathrm{ikl})+\exp (\mathrm{ikl})-2) \mathrm{p}_{0} \exp (-\mathrm{i} \omega \mathrm{t}+\mathrm{ikx})$

$13 \frac{\partial^{2} \mathrm{p}}{\partial \mathrm{r}_{2}^{2}}=0$

15 Using Eqs.(A.5) and (A.6), we can derive the dispersion relation of the mesh-free method in

case of $\mathrm{M}=2$ and $\mathrm{n}=8$. 
$2-\omega^{2}=\frac{v^{2}}{1^{2}}\{\exp (-\mathrm{ikl})+\exp (\mathrm{ikl})-2\}$

$3 \quad=-4 \frac{\mathrm{v}^{2}}{1^{2}} \sin ^{2}\left(\frac{\mathrm{kl}}{2}\right)$

$4 \omega=2 \frac{\mathrm{v}}{\mathrm{l}} \sin \left(\frac{\mathrm{kl}}{2}\right)$

5

$6 \quad$ The dispersion relations for different order and number of neighboring points can be derived

7 in a similar manner.

8 


\section{FIGURES}

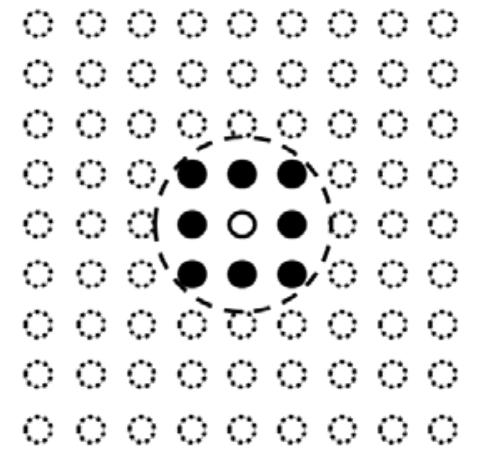

$$
M=2
$$

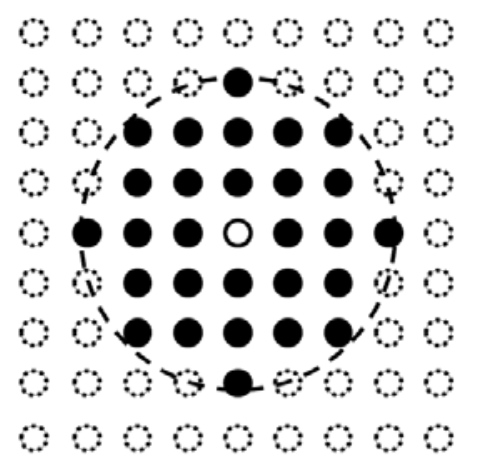

2

3 Figure 1. The choice of the influence domain for regular lattice alignment. Open solid circle

4 is the focusing point. Filled solid and open dotted circles represent calculation points inside

5 and outside the influence domain, respectively. Broken circle represents the influence

6 domain. The number of neighboring points is $8,20,28$ and 48 for $M=2,4,6$ and 8 , respectively.

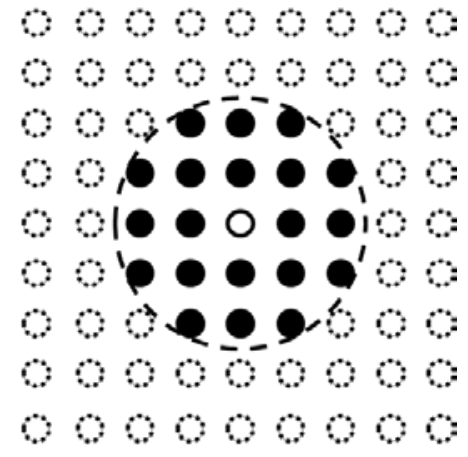

$M=4$

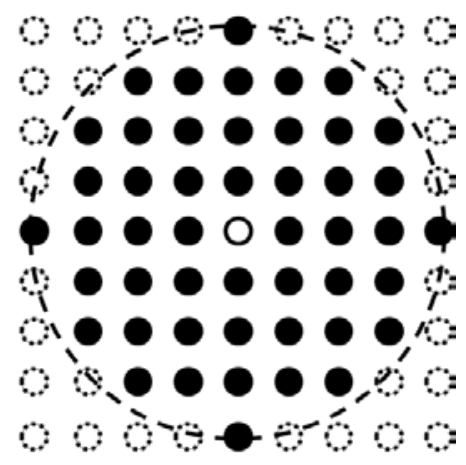

$\mathrm{M}=8$ 


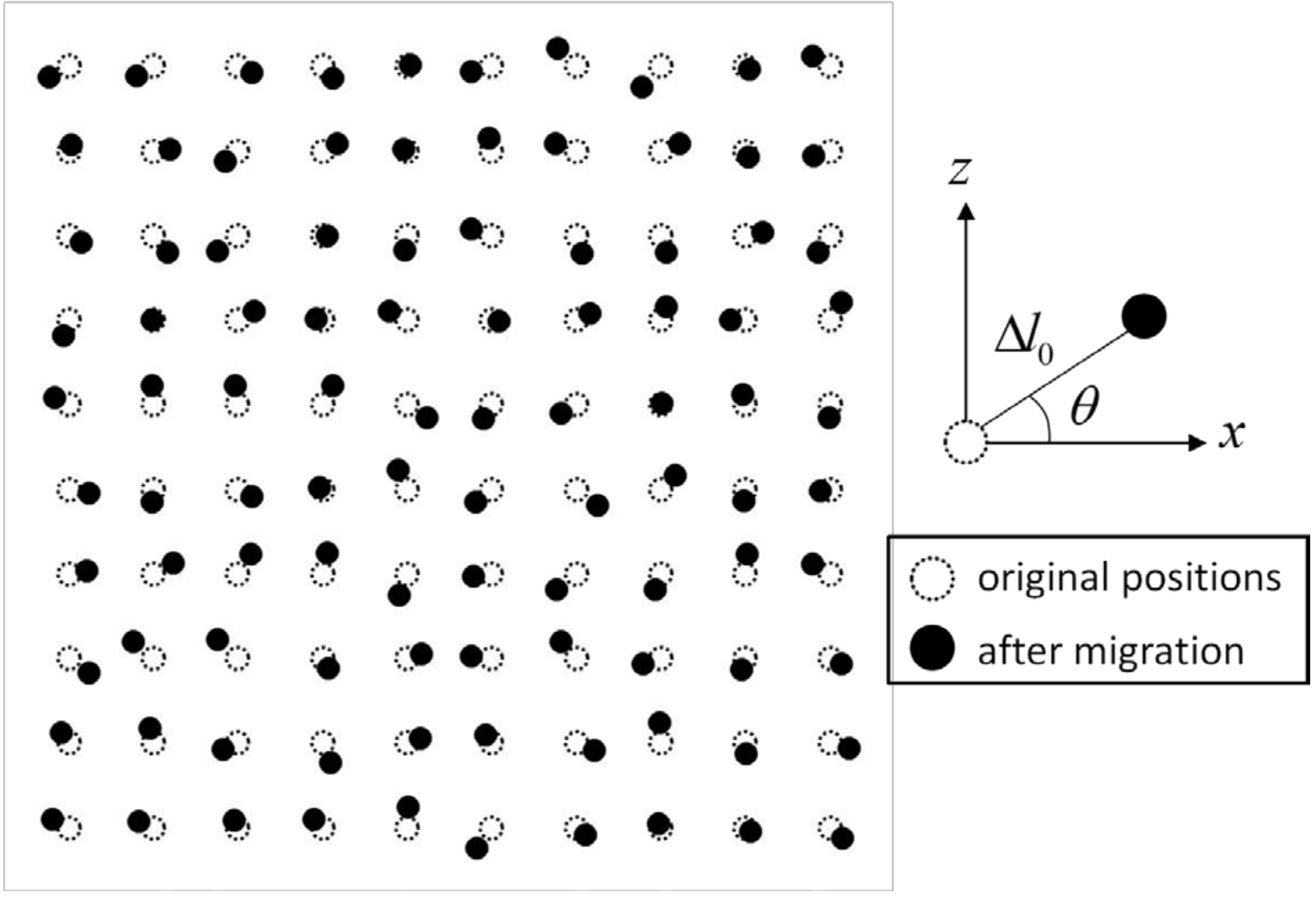

2 Figure 2. An example of irregular distribution of the calculation points. Each point is migrated in a random manner. 


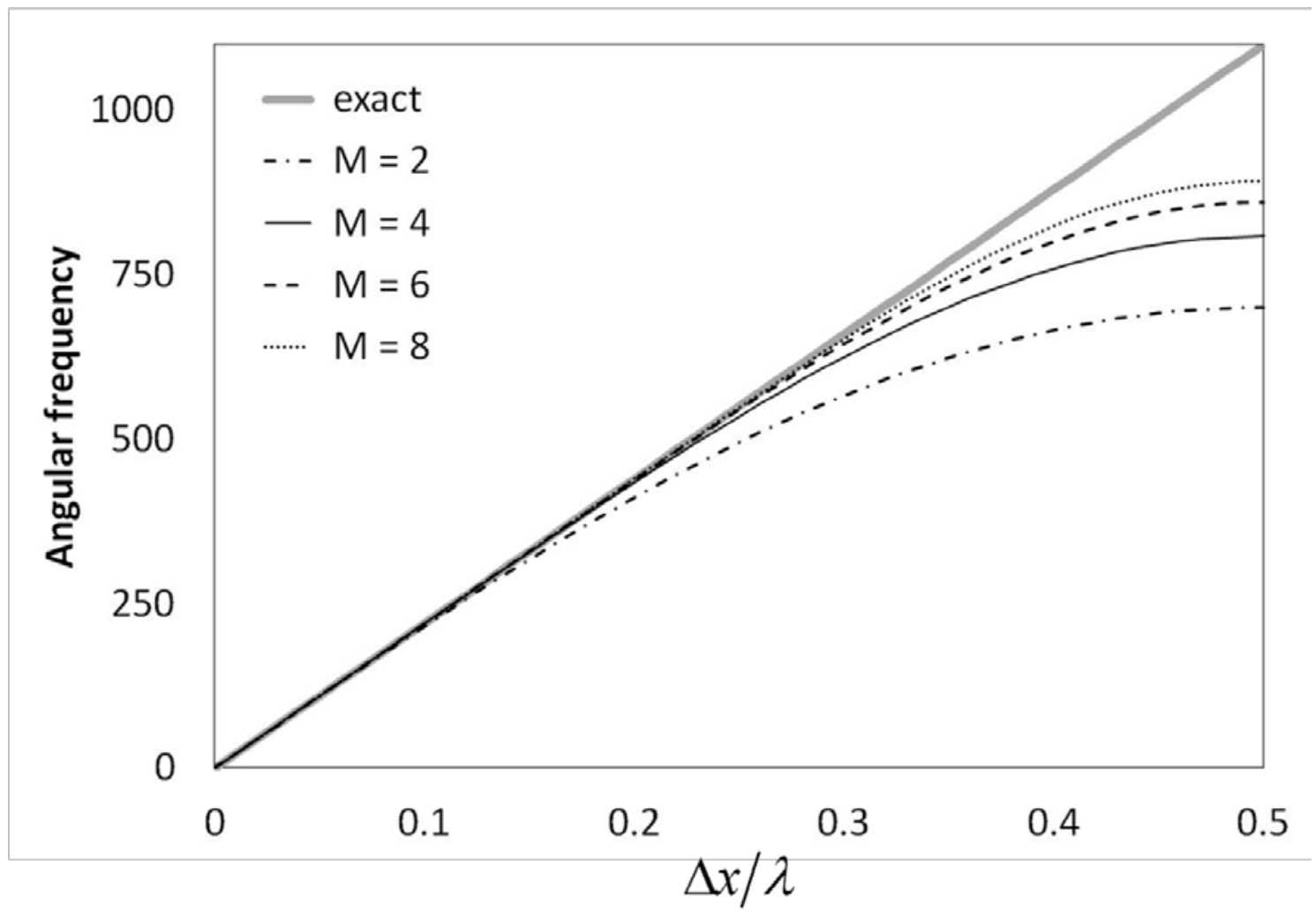

2 Figure 3. Dispersion curves for the regular arrangement with different order schemes. 

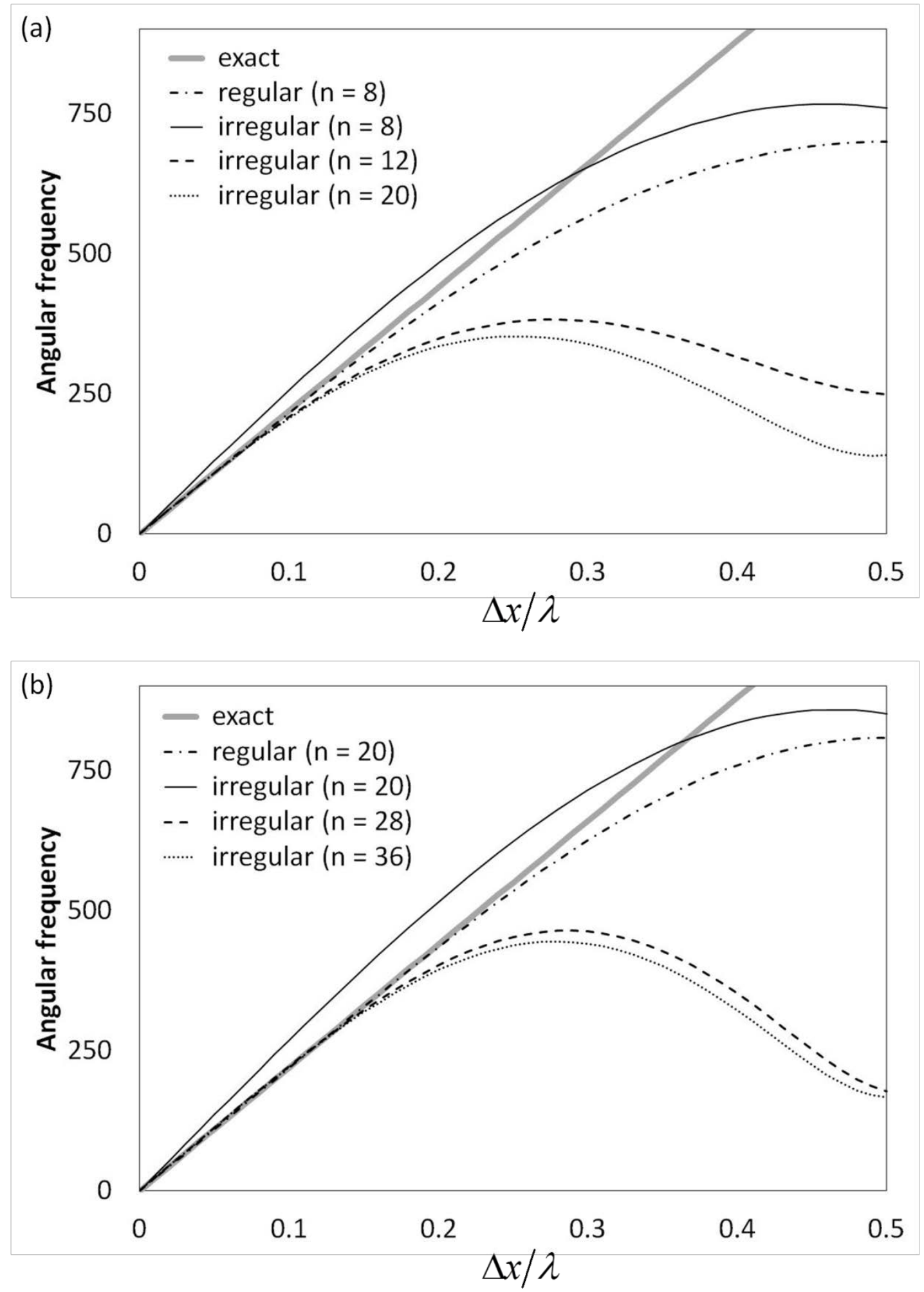

3 Figure 4. Dispersion curves for irregular arrangements with different number of neighbors.

$4 \quad$ (a) $M=2$, (b) $M=4$. 


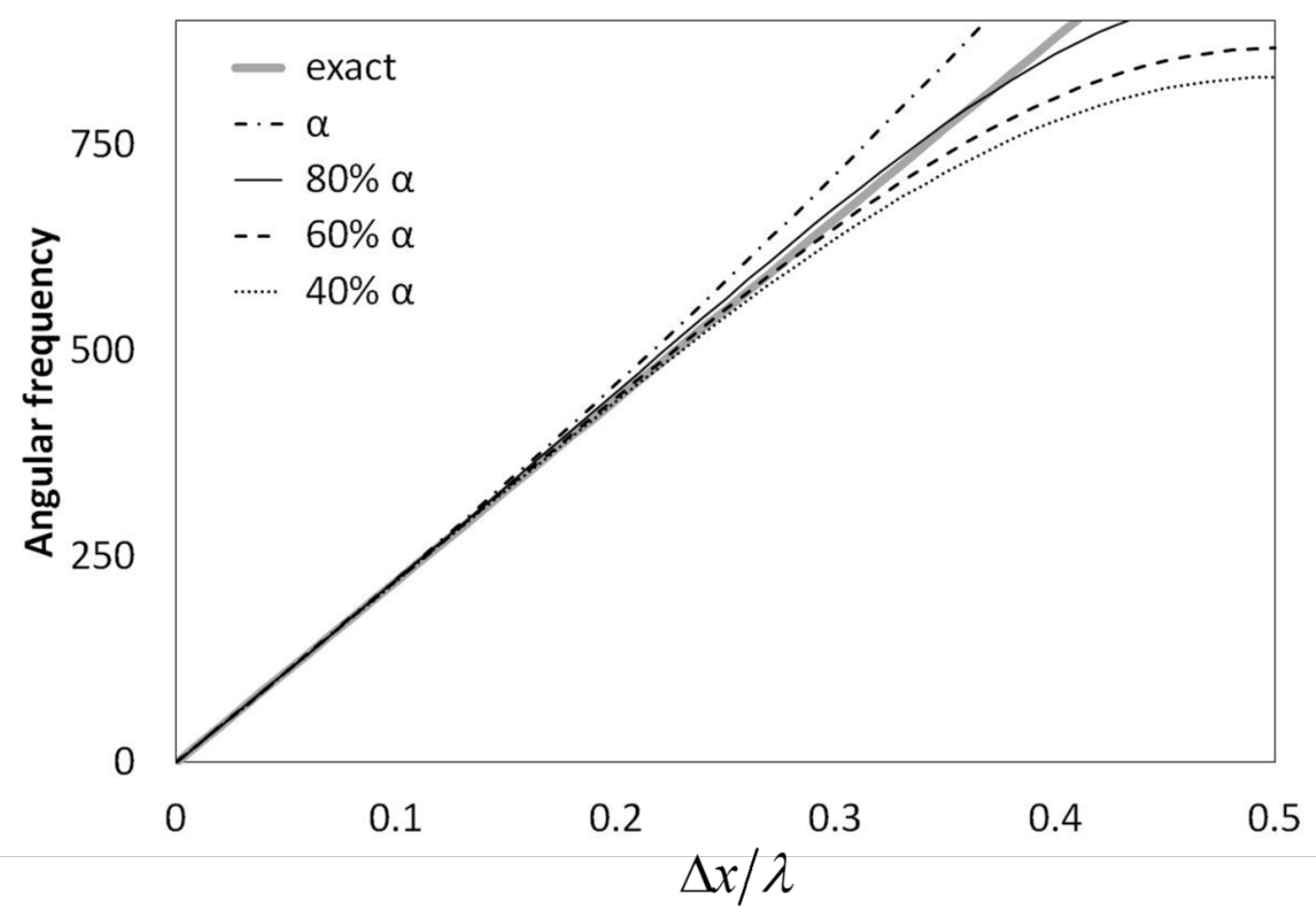

2 Figure 5. Dispersion curves with different time steps for $M=4 . \quad \alpha$ means the maximum

3 CFL number for stable calculations.

4 


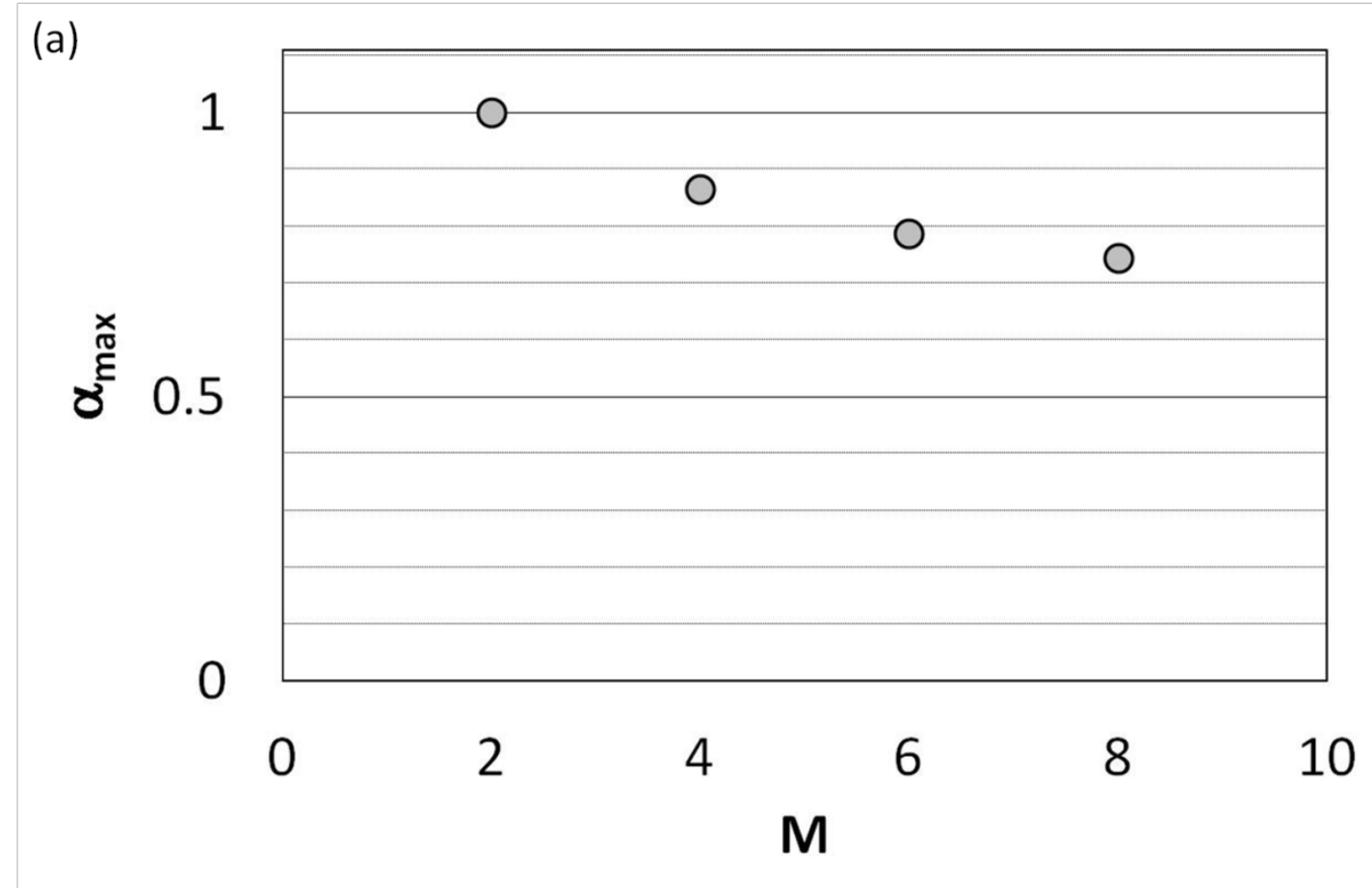

(b)

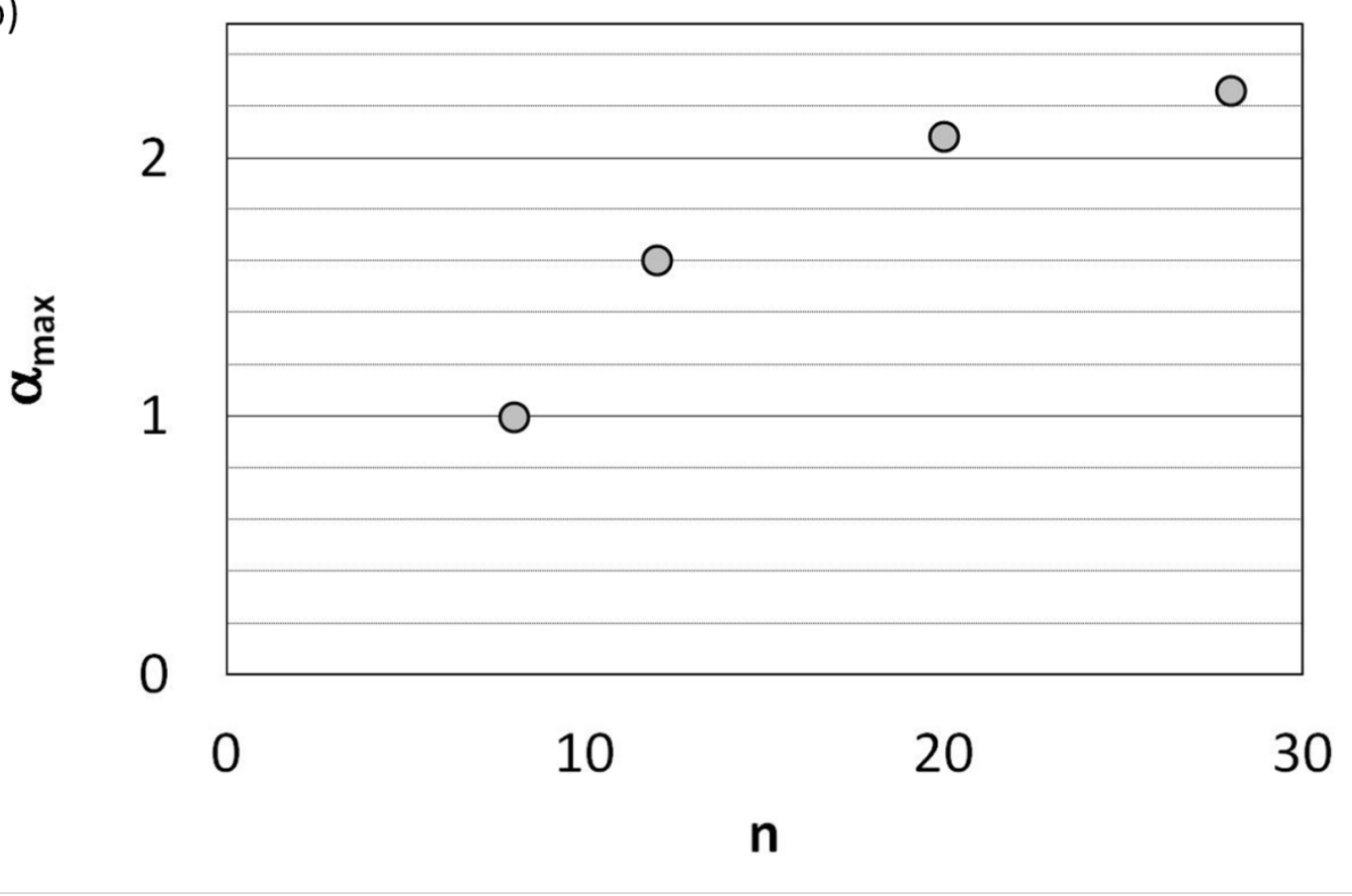

3 Figure 7. Stability condition of the mesh-free method with (a) different M, and (b) different n.

4 Vertical axis shows the maximum CFL number for stable calculation. 


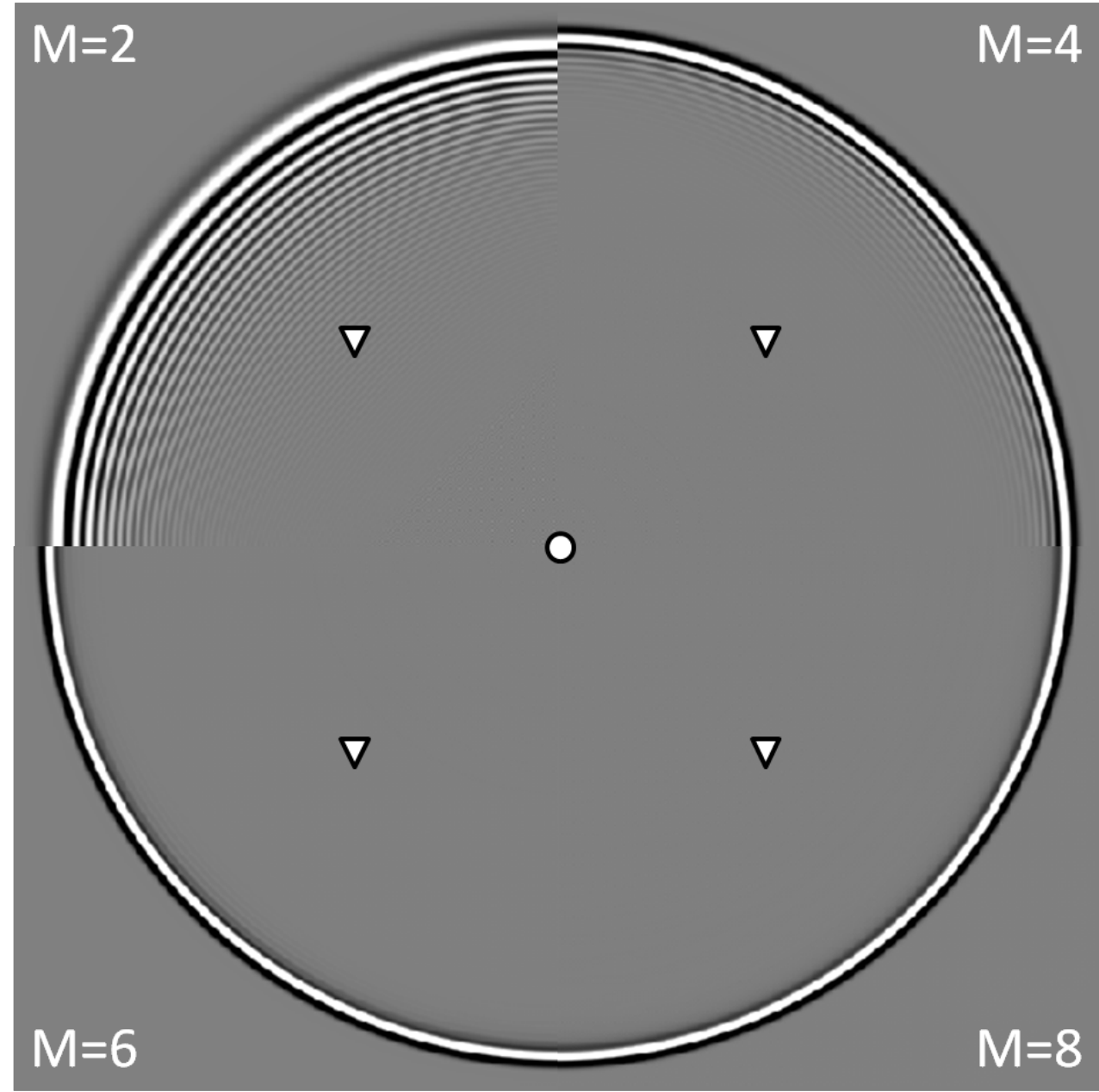

Figure 8. Snapshots of the pressure field with different order schemes after $1.75 \mathrm{~s}$. A circle and triangles represent source and receivers, respectively. 


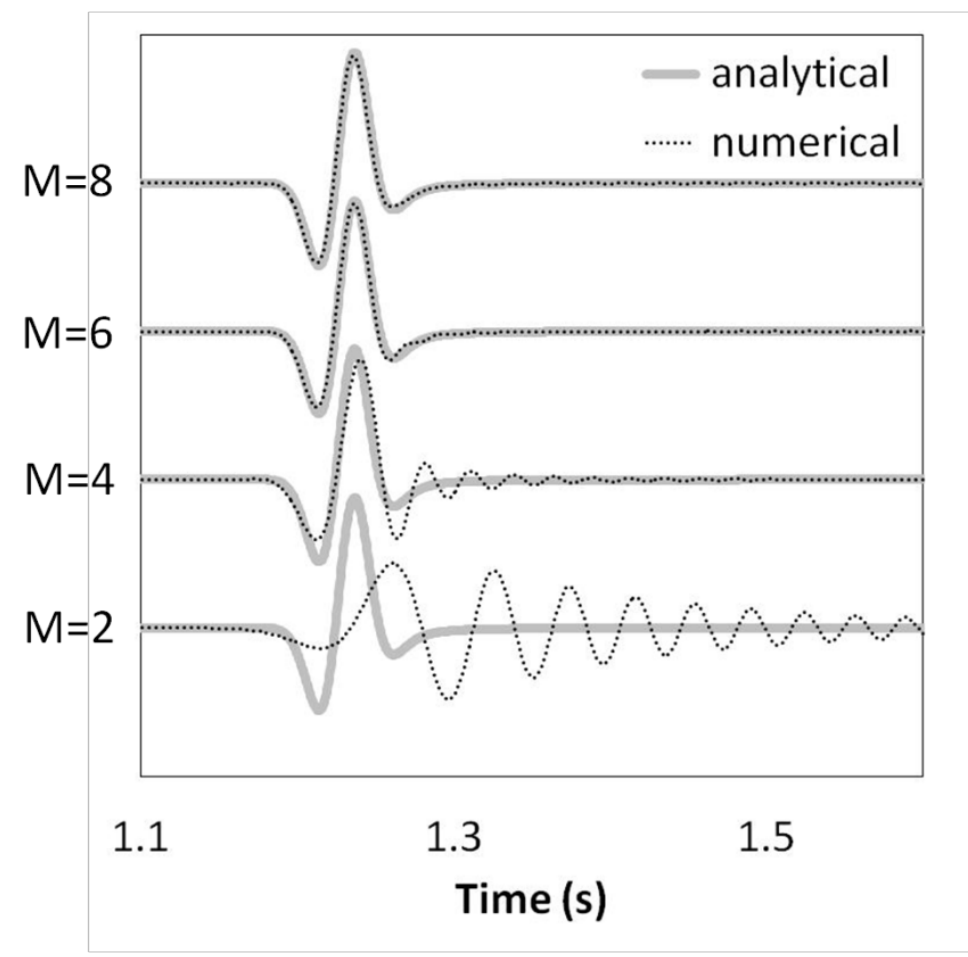

2 Figure 9. Waveforms observed at the receivers with different order schemes. Gray solid and

3 black dotted lines represent analytical and numerical waveforms, respectively.

4 


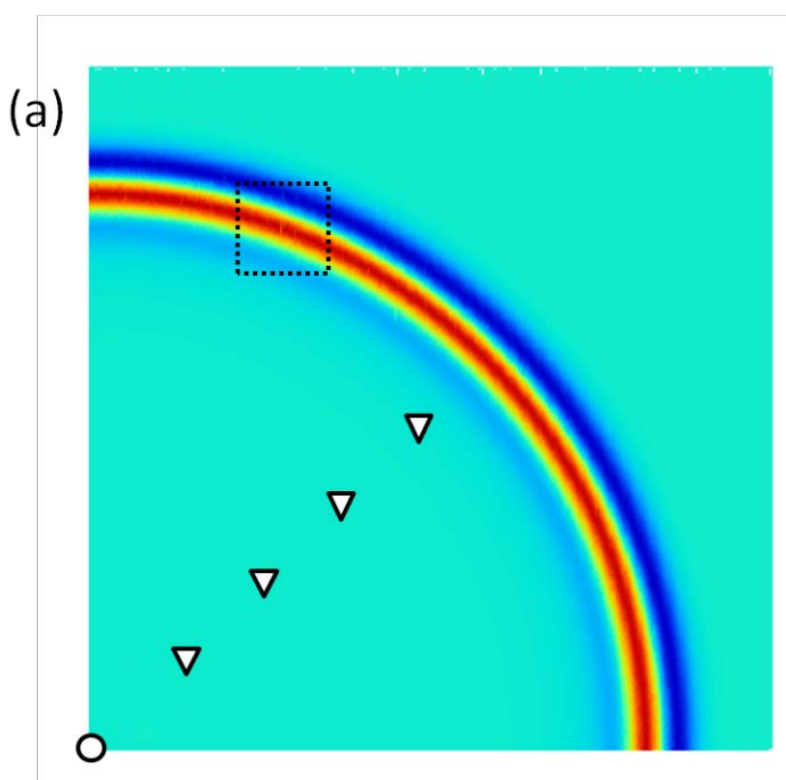

(b)

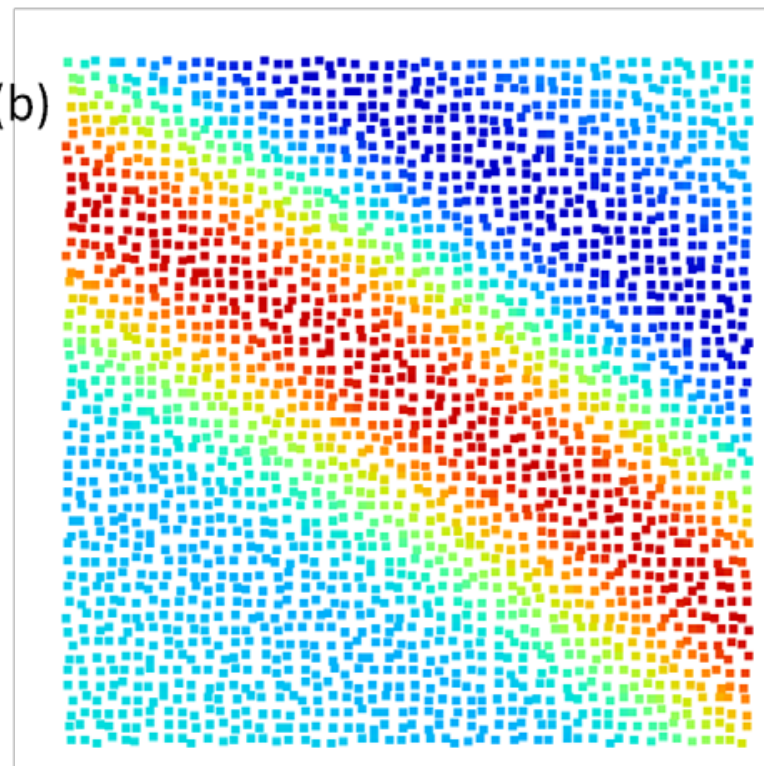

Figure 10. (a) A snapshot of the pressure field for an irregular arrangement. Open circle and

5 front. 


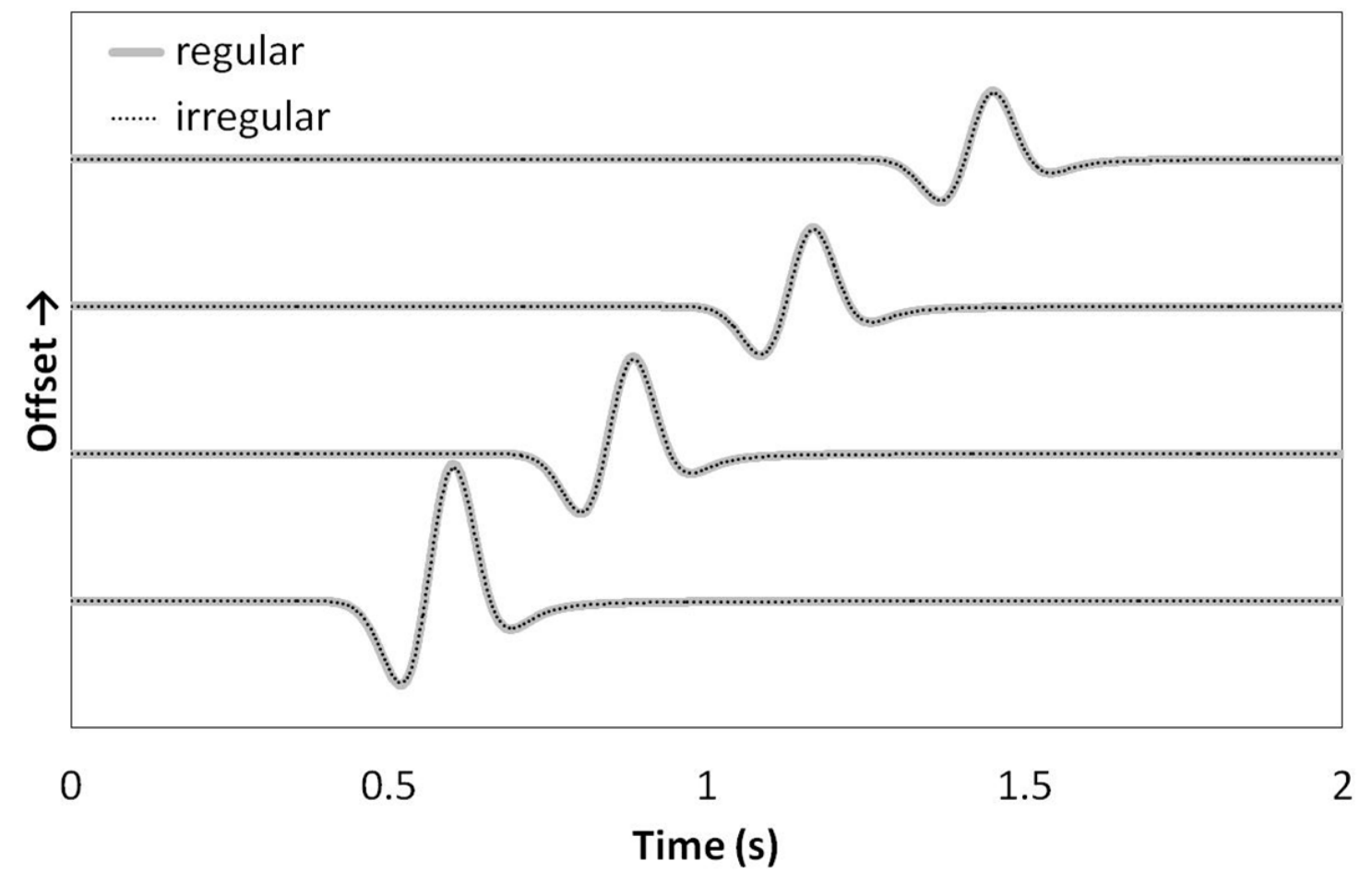

2 Figure 11. Waveforms obtained at the receivers with different offsets. Gray solid and black

3 dotted lines represent waveforms from regular and irregular arrangement, respectively. 


$$
V=2000 \mathrm{~m} / \mathrm{s}
$$

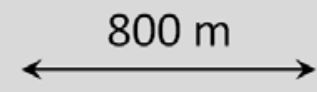

$V=1000 \mathrm{~m} / \mathrm{s}$

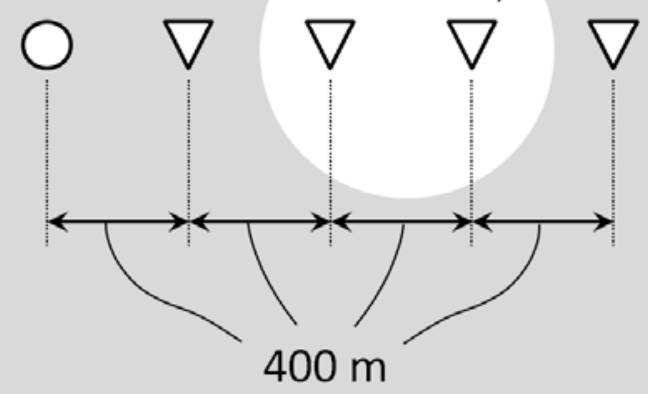

2 Figure 12. A schematic figure of an inhomogeneous model. A circular velocity anomaly,

3 whose diameter is $800 \mathrm{~m}$, is set at the center of the model. An open circle and triangles

4 represent the source and receivers. Four receivers are set with a constant offset of $400 \mathrm{~m}$. 
(a)

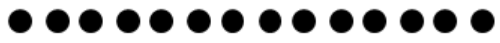

$\bullet \bullet \bullet \bullet \bullet \bullet \bullet \bullet \bullet \bullet \bullet \bullet \bullet \bullet$

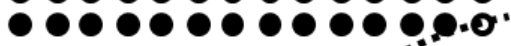

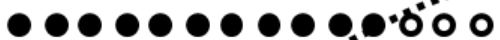

$\bullet \bullet \bullet \bullet \bullet \bullet \bullet \bullet \bullet \circ ० ० ०$

$\bullet \bullet \bullet \bullet \bullet \bullet .0 \% 000000$

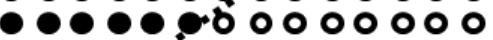

$\bullet \bullet \bullet .0^{\circ} 000000000$

$0 \bullet .00000000000$

- 4000000000000

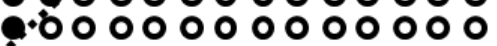

.00000000000000

00000000000000

00000000000000

(b)

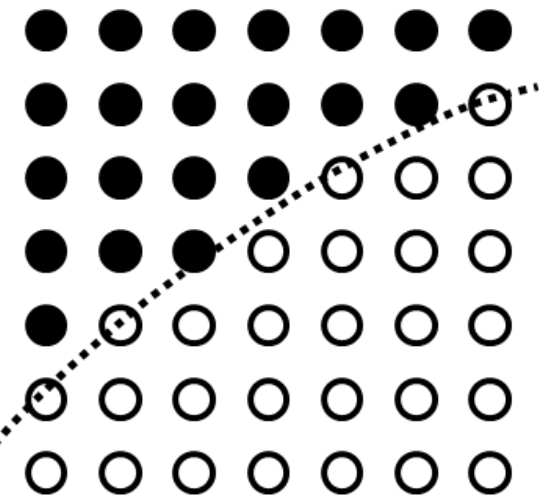

(c)

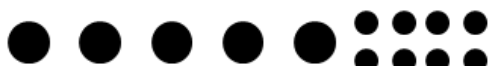

000000000.

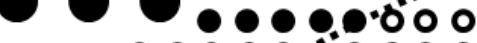

$000 \bullet 00000$

๑ Q.0\%0 00000

$0 \bullet \bullet .00000000$

0.0000000000

00.00000000000

0.000000000000

4.0000000000000

.000000000000000

100000000000000

00000000000000

Figure 13. Schematic figures of the distribution of calculation points around the interface of

5 the different spatial resolution. Open and filled circles represent the calculation points

6 belong to the anomaly and surrounding area, respectively. Dotted curve represents the

7 boundary between the anomaly and surrounding area, i.e. the lower and upper parts of the

8 dotted line are inside and outside of the anomaly, respectively. (a) fine arrangement, (b)

9 coarse arrangement, (c) partially fine arrangement. 
(a)

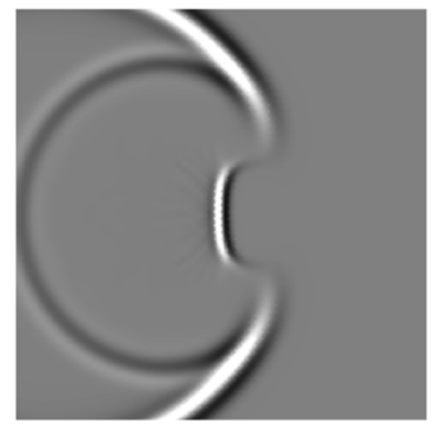

(b)

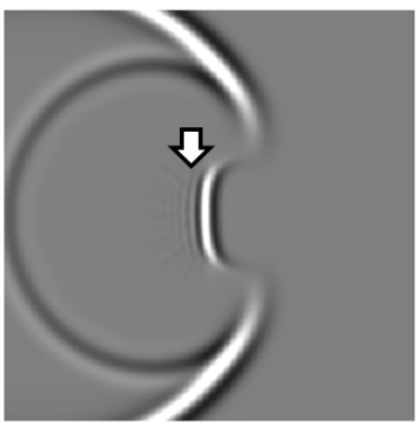

(d)

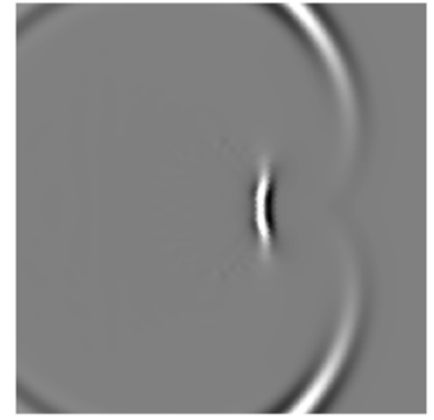

(e)

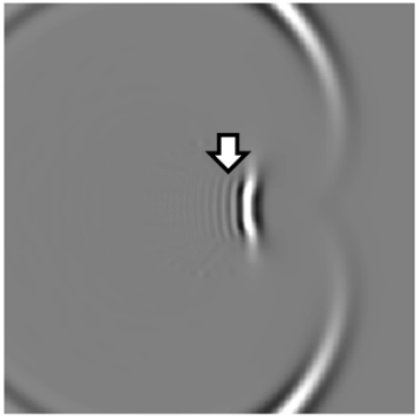

(c)

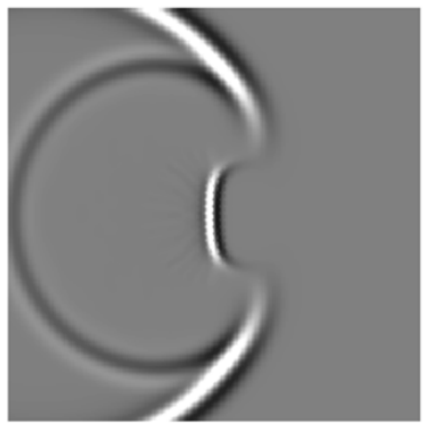

(f)

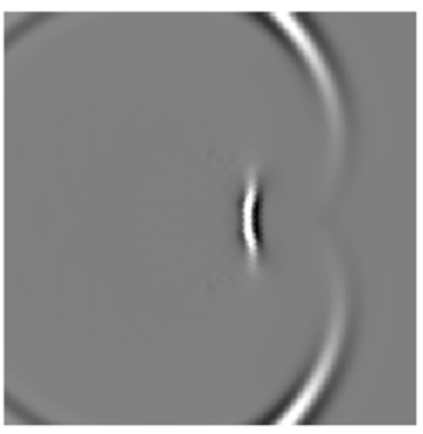

2 Figure 14. Snapshots of the pressure field after (a)-(c) $1 \mathrm{~s}$ and (d)-(f) $1.25 \mathrm{~s}$. (a) and (d) are

3 calculated by fine arrangement.

(b) and (e) are calculated by coarse arrangement.

(c) and

(f) are calculated by partially fine arrangement. 

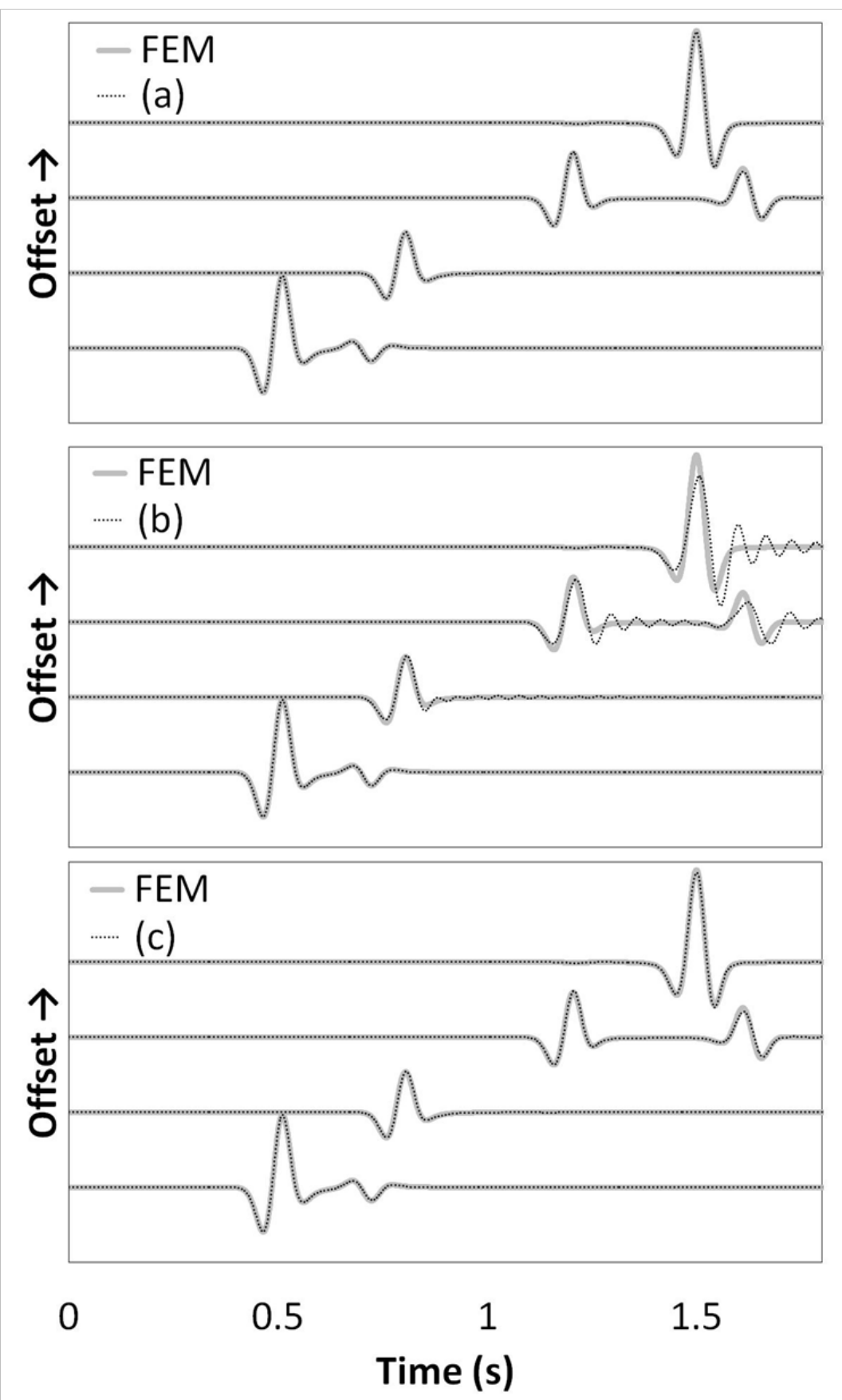

2 Figure 15. Waveforms obtained by different arrangement of calculation points compared with

3 FEM. Upper, middle and lower figures show the comparison between the fine, coarse,

4 partially fine arrangements and FEM, respectively. 

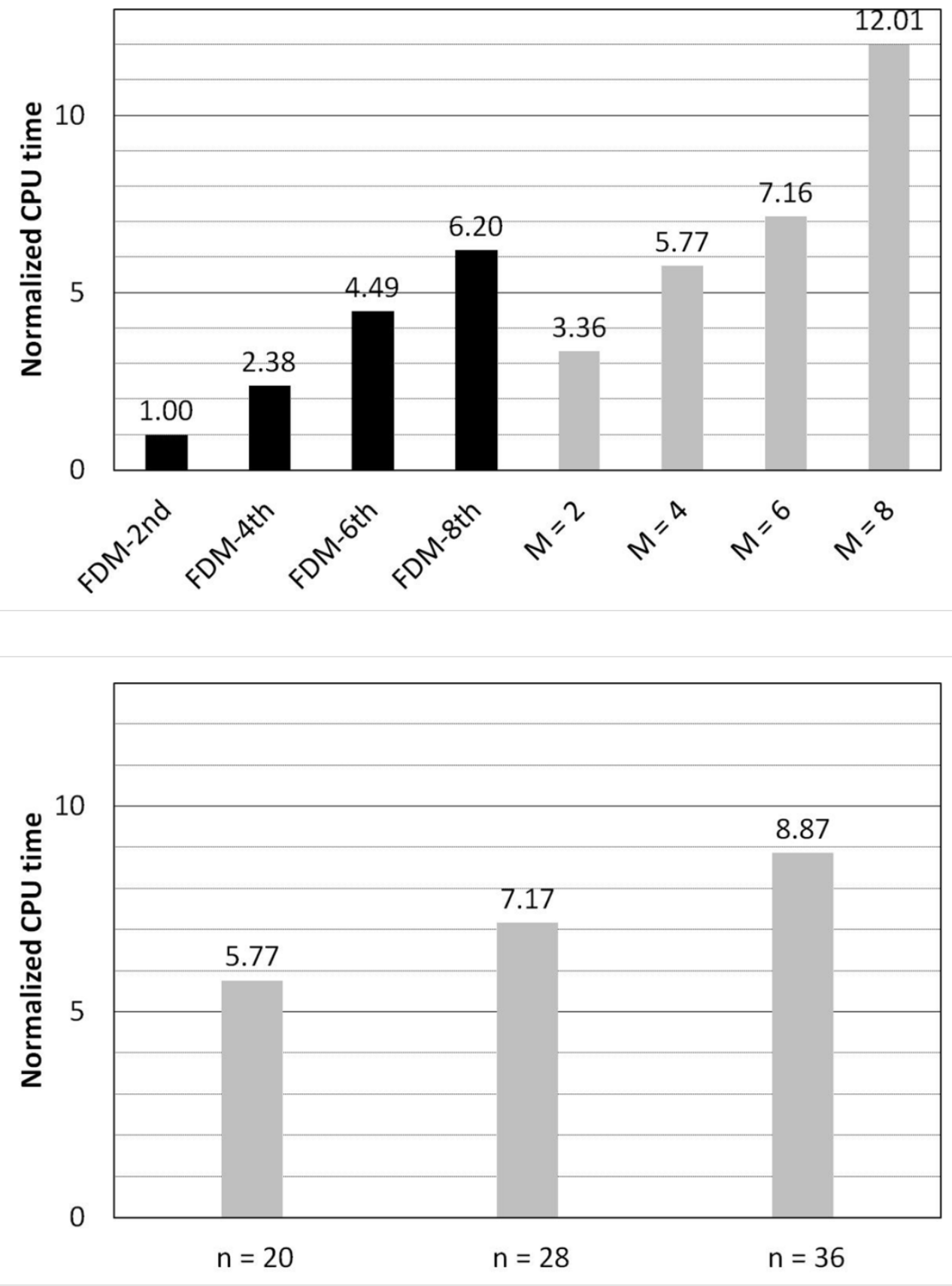

3 Figure 16. (a) Comparison of the calculation times of the mesh-free method with those of the

4 conventional FDM. (b) The effect of the number of calculation points on the neighboring

$5 \quad$ points for $\mathrm{M}=4$.

6 


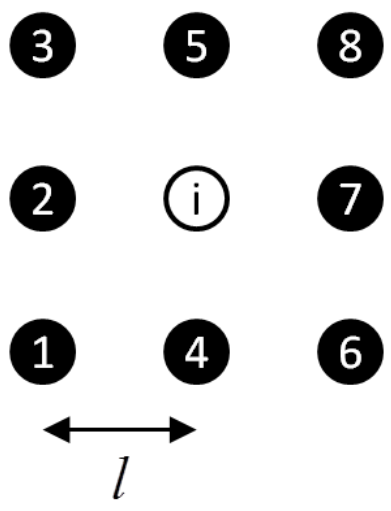

2 Figure A.1. Configuration of the target point $\mathrm{i}$ and neighboring points $\mathrm{j}(\mathrm{j}=1-8)$. $\quad \mathrm{l}$ means

3 the spacing of the regular lattice.

4 


\section{Acknowledgement}

2 We thank the editor Jef Caers, Gareth O'Brien and two anonymous reviewers for their

3 thoughtful comments and suggestions that improved the paper.

4

5 


\section{REFERENCES}

2 Aoi, S., and Fujiwara, H., 1999. 3D finite-difference method using discontinuous grids. Bull. Seismol. Soc. Am., 89, 918-930.

Cha, Y. H. and Shin, C., 2010. Two-dimensional Laplace-domain waveform inversion using adaptive meshes: an experience of the 2004 BP velocity-analysis benchmark data set. Geophys. J. Int., 182, 865-879. doi: 10.1111/j.1365-246X.2010.04618.x

Chen, J. B., 2011. A stability formula for Lax-Wendroff methods with forth-order in time and general-order in space for the scalar wave equation. Geophysics, 76, 2, T37-T42. doi: $10.1190 / 1.3554626$

Chen, J. B., 2013. A generalized optimal 9-point scheme for frequency-domain scalar wave equation, J. Appl. Geophys., 92, 1-7. doi: /10.1016/j.jappgeo.2013.02.008

Chu, C. and Stoffa, P. L., 2012. Determination of finite-difference weights using scaled binomial windows, Geophysics, 77, 3, W17-W26. doi: 10.1190/GEO2011-0336.1 element-free Galerkin method. Int. J. Num. Meth. Eng., 56, 331-350. doi: 10.1002/nme.564 acoustic finite-difference algorithms, Explor. Geophys., in press. doi: 10.1071/EG13072 
1 Lines, L. R., Slawinski, R., and Bording, R. P., 1999. A recipe for stability of finite-difference

2 wave-equation computations. Geophysics, 64, 3, 967-969.

3 Liu, C., Gao, F., Feng, X., Liu, Y., and Ren, Q., 2014a. Memoryless quasi-Newton (MLQN)

4 method for 2D acoustic full waveform inversion. Explor. Geophys., in press. doi: 10.1071/EG13090

Liu, H., Dai, N., Niu, F., and Wu, W., 2014b. An explicit time evolution method for acoustic wave propagation. Geophysics, 79, 3, T117-T124. doi: 10.1190/GEO2013-0073.1

Monette, L., and Anderson, M. P., 1994. Elastic and fracture properties of the

9 two-dimensional triangular and square lattices. Modelling Simul. Mater. Sci. Eng., 2, 53-66.

Nomura, K., Koshizuka, S., Oka, Y., and Obata, H., 2001. Numerical analysis of droplet breakup behavior using particle method. J. Nucl. Sci. Technol., 38, 1057-1064.

O’Brien, G., and Bean, C., 2004. A 3D discrete numerical elastic lattice method for seismic propagation in heterogeneous media with topography. Geophys. Res. Lett., 31, L14608. doi: 10.1029/2004GL020069

O’Brien, G. S., and Bean C. J., 2011. An irregular lattice method for elastic wave propagation. 
1 Saenger, E. H., N. Gold, and S. A. Shapiro, 2000. Modeling the propagation of elastic waves

$2 \quad$ using a modified fnite-difference grid. Wave Motion, 31, 77-92.

3 Saenger, E. H., and S. A. Shapiro, 2002. Effective velocities in fractured media: a numerical

4 study using the rotated staggered finite-difference grid. Geophys. Prospect., 50, 183-194.

5 Takekawa, J., Madariaga, R., Mikada, H., and Goto, T., 2012. Numerical simulation of

6 seismic wave propagation produced by earthquake by using a particle method. Geophys. J. Int., 191, 1305-1316. doi: 10.1111/j.1365-246X.2012.05676.X

Takekawa, J., Mikada, H., and Goto, T., 2014a. Numerical simulation using a Hamiltonian particle method for effective elastic properties in cracked media. Explor. Geophys., 45, 2, 116-124. doi:10.1071/EG13098

Takekawa, J., Mikada, H., and Goto, T., 2014b. A Hamiltonian particle method with a staggered particle technique for simulating seismic wave propagation. Pure Appl. Geophys., 171, 8, 1747-1757. doi: 10.1007/s00024-013-0763-x

Takekawa, J., Mikada, H., and Goto, T., 2014c. An accuracy analysis of a Hamiltonian particle method with the staggered particles for seismic-wave modeling including surface topography. Geophysics, 79, 4, T189-T197. doi: 10.1190/GEO2014-0012.1

Tamai, T., Shibata, K., and Koshizuka S., 2013. Development of the higher-order MPS method using the Taylor expansion, Trans. JSCES, Paper No.20130003. (in Japanese with 
$1 \quad$ English abstract)

2 Tan, S., and Huang, L., 2014. An efficient finite-difference method with high-order accuracy

3 in both time and space domains for modelling scalar-wave propagation. Geophys. J. Int., 197, 1250-1267. doi: 10.1093/gji/ggu077

Tarantola, A., 1984. Inversion of seismic reflection data in the acoustic approximation. Geophysics, 49, 1259-1266.

Tarrass, I., Giraud, L. and Thore, P. 2011. New curvilinear scheme for elastic wave propagation in presence of curved topography: Geophys. Prospect., 59, 889-906, doi: 10.1111/j.1365-2478.2011.00972.x. particle scheme. Geophys. J. Int., 141, 595-604. difference method. Geophysics, 51, 889-901.

14 Virieux, J., Calandra, H., and Plessix, R. E., 2011. A review of the spectral, pseudo-spectral, finite-difference and finite-element modeling techniques for geophysical imaging. Geophys. Prospect., 59, 794-813. doi: 10.1111/j.1365-2478.2011.00967.x 
1 interpolation method for the Helmholtz equation, Int. J. Num. Meth. Eng., 77, 1670-1689.

2 doi: $10.1002 /$ nme. 2463

3 Wenterodt, C., and Estorff, O., 2011, Optimized meshfree methods for acoustics, Comput.

$4 \quad$ Meth. Appl. Mech. Eng., 200, 2223-2236. doi: 10.1016/j.cma.2011.03.011

5 Wittke, J., and Tezkan, B., 2014. Meshfree magnetotelluric modelling, Geophys. J. Int., 198,

6 1255-1268. doi: 10.1093/gji/ggu207

$7 \quad$ Wu, W., Lines, L. R., and Lu, H., 1996. Analysis of higher-order finite difference schemes in

8 3-D reverse-time migration. Geophysics, 61, 845-856. 